\title{
Towards a unified theory of Sobolev inequalities
}

\author{
Joaquim Martín* \\ Department of Mathematics \\ Universitat Autònoma de Barcelona \\ jmartin@mat.uab.cat \\ Mario Milman ${ }^{\dagger}$ \\ Department of Mathematics \\ Florida Atlantic University \\ mario.milman@gmail.com \\ https://sites.google.com/site/mariomilman/
}

\section{Contents}

1 Introduction

2 Basic definitions and notation 2

3 Two Examples 3

4 Rearrangement invariant spaces and the Brezis-Wainger-Hansson embedding 5

5 Martin-Milman-Pustylnik meet Maz'ya 9

6 The Gaussian Inequality: Ledoux's inequality 9

7 The Metric Case 12

7.1 The Gagliardo-Nirenberg-Bobkov-Houdre inequality . . . . . . . 12

7.2 Generalized Pólya-Szegö . . . . . . . . . . . . . . . . . . . . 13

7.3 Maz'ya-Talenti inequality . . . . . . . . . . . . . . . 15

7.4 Basic rearrangement inequality . . . . . . . . . . . . . 15

7.5 Self Improvement . . . . . . . . . . . . . . . . . . . . . . 16

7.6 Poincaré inequalities and generalized Pólya-Szegö . . . . . . . . . 16

7.7 Identity associated with Poincaré . . . . . . . . . . . . . 18

*Partially supported in part by Grants MTM2010-14946, MTM-2010-16232

${ }^{\dagger}$ Partially supported by a grant from the Simons Foundation (\#207929 to Mario Milman). 
8 Coulhon Inequalities 18

9 Connection with the work of Emanuel Milman 21

9.1 Isoperimetric Hardy type . . . . . . . . . . . . . . 22

10 Transference and dimensionless inequalities 23

11 Rearrangement inequalities of Garsia-Rodemich type and Morrey's theorem $\quad 26$

12 Higher Order Inequalities 3

\begin{abstract}
We discuss our work on pointwise inequalities for the gradient which are connected with the isoperimetric profile associated to a given geometry. We show how they can be used to unify certain aspects of the theory of Sobolev inequalities. In particular, we discuss our recent papers on fractional order inequalities, Coulhon type inequalities, transference and dimensionless inequalities and our forthcoming work on sharp higher order Sobolev inequalities that can be obtained by iteration.
\end{abstract}

\title{
1 Introduction
}

In this expository note we survey some of our previous work towards a unified theory of Sobolev inequalities using pointwise rearrangement inequalities. The presentation includes our recent results [80] on Coulhon inequalities (cf. [28, [5]), integral transference and dimensionless inequalities [81, fractional inequalities [79, extrapolation and self improvement [78. We also include a brief section on how iterations of our estimates lead to sharp higher order pointwise inequalities 82 . We hope that this attempt to summarize and organize some of the material, together with the inclusion of our motivation and details on the methods used, could be of some interest.

In conclusion we should mention three important lessons we learned from Gian Carlo Rota [106:

1. Publish the same results several times 2. Do not worry about your mistakes and 3. Write informative introductions.

\section{Basic definitions and notation}

Before start our presentation let us recall some basic definitions and set up the notation.

Let $(\Omega, \mu)$ be a measure space $(\Omega, \mu)$; for a measurable function $u: \Omega \rightarrow \mathbb{R}$, the distribution function of $u$ is given by

$$
\mu_{u}(t)=\mu\{x \in \Omega:|u(x)|>t\} \quad(t \geq 0) .
$$


The decreasing rearrangement of a function $u$ is the right-continuous nonincreasing function from $[0, \mu(\Omega))$ into $\mathbb{R}$ which is equimeasurable with $u$. It can be defined by the formula

$$
u_{\mu}^{*}(s)=\inf \left\{t \geq 0: \mu_{u}(t) \leq s\right\}, \quad s \in[0, \mu(\Omega)) .
$$

The maximal average $u_{\mu}^{* *}(t)$ is defined by

$$
u_{\mu}^{* *}(t)=\frac{1}{t} \int_{0}^{t} u_{\mu}^{*}(s) d s
$$

When the measure is clear from the context, or when we are dealing with Lebesgue measure, we may simply write $u^{*}$ and $u^{* *}$, etc.

Let $X=X(\Omega)$ be a Banach function space on $(\Omega, \mu)$, with the Fatou property 1 . We shall say that $X$ is a rearrangement-invariant (r.i.) space, if $g \in X$ implies that all $\mu$-measurable functions $f$ with $f_{\mu}^{*}=g_{\mu}^{*}$, also belong to $X$ and moreover, $\|f\|_{X}=\|g\|_{X}$. The functional $\|\cdot\|_{X}$ will be called a rearrangement invariant norm. Typical examples of r.i. spaces are the $L^{p}$-spaces, Orlicz spaces, Lorentz spaces, Marcinkiewicz spaces, etc.

Let $X(\Omega)$ be a r.i. space, then there exists a r.i. space $\bar{X}=\bar{X}(0, \mu(\Omega))$ on $((0, \mu(\Omega)), m),(m$ denotes the Lebesgue measure on the interval $(0, \mu(\Omega)))$ such that

$$
\|f\|_{X(\Omega)}=\left\|f_{\mu}^{*}\right\|_{\bar{X}(0, \mu(\Omega))} \cdot
$$

$\bar{X}$ is called the representation space of $X(\Omega)$ which is unique if $\mu(\Omega)$ is finite.) We refer the reader to [15] for a complete treatment of the theory of (r.i.) spaces.)

As usual, the symbol $f \simeq g$ will indicate the existence of a universal constant $c>0$ (independent of all parameters involved) so that $(1 / c) f \leq g \leq c f$, while the symbol $f \preceq g$ means that $f \leq c g$, finally given $1 \leq p \leq \infty, p^{\prime}=p /(p-1)$.

\section{Two Examples}

We state two simple examples that illustrate the difficulties in deriving a unified theory of Sobolev inequalities.

Example 1 In dimension 1 the Sobolev embedding theorem is connected with the fundamental theorem of calculus: If $f$ is a Lip function with compact support, then

$$
f(y)=\int_{-\infty}^{y} f^{\prime}(s) d s
$$

\footnotetext{
${ }^{1}$ This means that if $f_{n} \geq 0$, and $f_{n} \uparrow f$, then $\left\|f_{n}\right\|_{X} \uparrow\|f\|_{X}$ (i.e. Fatou's Lemma holds in the $X$ norm).
} 
and therefort2

$$
\|f\|_{\infty} \leq\left\|f^{\prime}\right\|_{1} .
$$

In dimension $n$ the corresponding estimate does not hold. In particular, there are functions $f \in W^{1, n}\left(\mathbb{R}^{n}\right)$ that are not bounded. Instead, we have somewhat weaker results: (Sobolev)

$$
\|f\|_{p_{n}} \leq c_{n, p}\|\nabla f\|_{p}, f \in C_{0}^{\infty}\left(\mathbb{R}^{n}\right), \text { with } \frac{1}{p_{n}}=\frac{1}{p}-\frac{1}{n}, 1<p<n,
$$

with $c_{n} \rightarrow \infty$, when $p \rightarrow n$. The inequality is also true when $p=1$ (GagliardoNirenberg): there exists $c_{n}$ such that

$$
\|f\|_{n^{\prime}} \leq c_{n}\|\nabla f\|_{1}, f \in C_{0}^{\infty}\left(\mathbb{R}^{n}\right) .
$$

One way to complete the picture for $p=n$, is by extrapolation: Trudinger [112] showed by extrapolation that if $\Omega$ is an open set in $\mathbb{R}^{n}$ with $|\Omega|<\infty$, then

$$
W_{0}^{1, n}(\Omega) \subset e^{L^{n^{\prime}}} .
$$

This example, in particular, illustrates the crucial role of dimension in the Euclidean Sobolev inequalities.

As a counterpart, consider the following

Example 2 Consider $\mathbb{R}$ but now equipped with one dimensional Gaussian measure $\gamma_{1}$. In this case the gain of integrability is logarithmic ("logarithmic type Sobolev inequalities")

$$
\|f\|_{L(\log L)^{1 / 2}\left(\gamma_{1}\right)} \leq c\left(\|\nabla f \mid\|_{L^{1}\left(\gamma_{1}\right)}+\|f\|_{L^{1}\left(\gamma_{1}\right)}\right) .
$$

Similarly, for $p>1$ we have

$$
\|f\|_{L^{p}(\log L)^{p / 2}\left(\gamma_{1}\right)} \leq c\left(\||\nabla f|\|_{L^{p}\left(\gamma_{1}\right)}+\|f\|_{L^{p}\left(\gamma_{1}\right)}\right) .
$$

Moreover, the gain of integrability does not change with the dimension! For example, for $\mathbb{R}^{n}$ equipped with Gaussian measure $\gamma_{n}$, we have

$$
\|f\|_{L(\log L)^{1 / 2}\left(\gamma_{n}\right)} \leq c\left(\||\nabla f|\|_{L^{1}\left(\gamma_{n}\right)}+\|f\|_{L^{1}\left(\gamma_{n}\right)}\right) .
$$

In other words, while in the Euclidean geometry the spaces involved in the inequalities depend on the dimension, and the gain of integrability is naturally measured with powers, i.e. using $L^{p}$ or $L(p, q)$ spaces, in the Gaussian world the gain of integrability is logarithmic, independent of the dimension, and the right spaces one needs to measure the gain of integrability are logarithmic Orlicz type spaces. The celebrated logarithmic Sobolev inequalities of Gross (cf. [47]) are among the most prominent examples of dimensionless inequalities in the Gaussian world (cf. [47]).

\footnotetext{
2 actually using the available cancellation, we have $2 f(y)=\int_{-\infty}^{y} f^{\prime}(s) d s+\int_{y}^{\infty} f^{\prime}(s) d s$, therefore the constant of the embedding can be improved,

$$
\|f\|_{\infty} \leq \frac{1}{2}\left\|f^{\prime}\right\|_{1} .
$$
}


Traditionally this state of affairs has led to different methods/theories to deal with Euclidean or Gaussian Sobolev inequalities, or more generally, Sobolev inequalities in other geometries, e.g. Euclidean domains with measures of the form $w(x) d x$, Riemannian manifolds, or more generally, metric measure spaces. In particular, what is the role of dimension? What are the function spaces one needs to use to measure the integrability gains?

While some of the differences are unavoidable, one wonders if it is possible to unify at least some aspects of these disparate theories and thus, maybe, provide a better understanding. Recently there has been progress in this direction from several different directions by a number of authors. The results presented in this note describe mainly of our work towards a unified theory of Sobolev inequalities using pointwise inequalities on rearrangements; as consequence the list of references is disproportionately tilted towards our own work. Moreover, there is a huge literature on Sobolev inequalities, and while the bibliography we have compiled is rather large, we must warn the reader that we did not attempt to cover all the references, not even the important references. We apologize in advance if your favorite paper/author is not included in the bibliography, hopefully it should not be more than one (reference) iteration away. In particular, we should explicitly mention our debt to the pioneering 3 work of V. Maz'ya which has greatly influenced our view point of the subject (cf. Section 5 below). For more information, background and more comprehensive bibliographies we refer to [1, 34, 49], 68, [77, 87, [101, 104, [107, and [110.

\section{Rearrangement invariant spaces and the Brezis- Wainger-Hansson embedding}

One early difficulty in trying to develop any type of unified theory is that, even in the Euclidean case, the limiting borderline case $(p=n)$ of the Sobolev embedding apparently requires the use of a different scale of spaces, e.g. an "extrapolation space"...So we started our work trying to understand the issues connected with the limiting inequalities. In a convoluted way our efforts to understand the limiting cases eventually led us to a better understanding on how to approach all the cases simultaneously! point where to start with the story.

An early result in this direction was obtained by Trudinger [112, who showed that for a domain $\Omega$ in $\mathbb{R}^{n}$, with $|\Omega|<\infty$,

$$
W_{0}^{1, n}(\Omega) \subset e^{L^{n^{\prime}}}(\Omega) \text {. }
$$

\footnotetext{
${ }^{3}$ For a detailed presentation of Maz'ya's remarkable early work we refer to [51.

${ }^{4}$ This is a somewhat dissapointing turn of events for the developers of general abstract theories studying limiting inequalities (e.g. [55]) but our current understanding of Sobolev inequalities shows that: (a) Sobolev inequalities self improve (cf. [5, 48, 85, 78, ) and (b) the extrapolations of Sobolev inequalities take the form: "one inequality" implies a family of inequalities and in some cases "one inequality implies all" !(cf. [5], 29], 78])). We also refer to the forthcoming 83 for a connection with extrapolation of Sobolev inequalities à la Rubio de Francia.
} 
Brezis-Wainger [23] improved this result using the rearrangement inequalities of O'Neil [99], while Hansson [50] obtained similar results. Their result reads

$$
W_{0}^{1, n}(\Omega) \subset B W H(\Omega),
$$

where if, say, $|\Omega|=1$, then $B W H=$ (Brezis-Wainger-Hansson) is the space defined by

$$
\|f\|_{B W H}=\left\{\int_{0}^{1}\left(f^{* *}(s) \frac{1}{1+\log \frac{1}{s}}\right)^{n} \frac{d s}{s}\right\}^{1 / n}<\infty .
$$

Maz'ya (cf. [87, (3.1.4) page 232] and the references therein) had obtained earlier an inequality using his capacity theory that, in particular, implies the embedding (3). The improvement over (2) is given by the easily verified fact that

$$
B W H(\Omega) \subset e^{L^{n^{\prime}}}(\Omega) .
$$

On the other hand, O'Neil [99] extended the original (one dimensional) results of Hardy-Littlewood, as follows

$$
W^{1, p}\left(\mathbb{R}^{n}\right) \subset L\left(p_{n}, p\right), 1<p<n, \frac{1}{p_{n}}=\frac{1}{p}-\frac{1}{n},
$$

where the Lorentz $L(p, q)$ spaces, for $p<\infty, q \in[1, \infty]$ are defined by

$$
L(p, q)=\left\{f:\|f\|_{L(p, q)}=\left\{\int_{0}^{\infty}\left(f^{* *}(t) t^{1 / p}\right)^{q} \frac{d t}{t}\right\}^{1 / q}<\infty\right\} .
$$

The results of O'Neil and Brezis-Wainger-Hansson, like the original one dimensional results of Hardy-Littlewood, can be shown to be optimal within the class of rearrangement invariant spaces: if $X$ is a rearrangement invariant space then,

$$
W^{1, p} \subset X \Rightarrow L\left(p_{n}, p\right) \subset X \text {, if } p<n \text {, or } B W H \subset X \text {, if } p=n \text {. }
$$

As a consequence, the $L(p . q)$ spaces are not enough to describe the optimal Sobolev inequalities (e.g. the case $p=n$ requires a different space). This led to the introduction in the theory of Sobolev embeddings of the following modification of the $L(p, q)$ spaces which, as we shall see, resolves this difficulty (cf. [10, 11] and, as it turns out, in a different way and less explicitly in [111] and [69]). For a measure space we define

$$
L(\infty, \infty)=\left\{f:\|f\|_{L(\infty, \infty)}=\sup _{t}\left(f^{* *}(t)-f^{*}(t)\right)<\infty\right\} .
$$

This space was introduced by Bennett-DeVore-Sharpley [14 who in their paper show that for functions defined on a cube, $L(\infty, \infty)$ is the rearrangement invariant hull of $B M O$, i.e. $L(\infty, \infty)$ is the smallest possible space that contains all the rearrangements of functions in $B M O$. One should note here that the usual definition of $L(\infty, \infty)$ would give

$$
\|f\|_{L(\infty, \infty)}=\sup _{t} f^{* *}(t)=\|f\|_{L^{\infty}},
$$


while the space $L(\infty, \infty)$ that we have defined is bigger: we have $B M O \subset$ $L(\infty, \infty)$.

More generally, note that if we formally attempt to define the $L(\infty, q)$ spaces using the classical definition the resulting spaces are trivial:

$$
\int_{0}^{\infty} f^{* *}(s)^{q} \frac{d s}{s}<\infty \Rightarrow f=0 .
$$

On the other hand, if we redefine the $L(\infty, q)$ spaces by means of replacing $f^{* *}(t)$ by the oscillation $f^{* *}(t)-f^{*}(t)$, then the spaces defined by the condition

$$
\|f\|_{L(\infty, q)}=\left\{\int_{0}^{|\Omega|}\left(\left(f^{* *}(t)-f^{*}(t)\right)\right)^{q} \frac{d t}{t}\right\}^{1 / q}<\infty,
$$

are not trivial, since the differentiation theorem provides us with a cancellation at the origin. In [10, 11] the role of the $L(\infty, q)$ spaces in the Sobolev embedding was observed. It was noted there that these spaces were relevant in connection with an inequality implicit in the paper by Alvino-Trombetti-Lions [4]: If $f$ is symmetrically decreasing, then

$$
f^{* *}(t)-f^{*}(t) \leq c_{n} t^{1 / n}|\nabla f|^{* *}(t) .
$$

Now, if we let $f^{\circ}$ denote the symmetric rearrangement of $f$ (cf. [60, 68]), then for smooth $f$, the Pólya-Szegö principle can be formulated as (cf. [85] and the references there to earlier work by Fournier)

$$
\left|\nabla f^{\circ}\right|^{* *}(t) \leq|\nabla f|^{* *}(t) .
$$

Then, since $\left(f^{\circ}\right)^{*}=f^{*}$, it follows that we can eliminate the restriction for $f$ to be symmetrically decreasing, therefore (4), indeed, holds for all smooth $f$. Next, integrating (4), we see that:

$$
\left\{\int_{0}^{|\Omega|}\left(\left(f^{* *}(t)-f^{*}(t)\right) t^{1 / p-1 / n}\right)^{q} \frac{d t}{t}\right\}^{1 / q} \leq c_{n}\left\{\int_{0}^{|\Omega|}\left(t^{1 / p}|\nabla f|^{* *}(t)\right)^{q} \frac{d t}{t}\right\}^{1 / q} .
$$

The left hand side is equivalent to $\|f\|_{L\left(p_{n}, q\right)}$ (cf. [72]), and moreover, for $q=p$ we have

$$
\left\{\int_{0}^{|\Omega|}\left(t^{1 / p}|\nabla f|^{* *}(t)\right)^{p} \frac{d t}{t}\right\}^{1 / p} \leq p^{\prime}\||\nabla f|\|_{p} .
$$

Thus, for $q=p<n$ we have recovered the classical Sobolev inequality. Moreover, the inequality (5) is valid and makes sense in the limiting case $p=n$. In particular for $q=p=n$, we have

$$
\left\{\int_{0}^{|\Omega|}\left(\left(f^{* *}(t)-f^{*}(t)\right)\right)^{n} \frac{d t}{t}\right\}^{1 / n} \leq c_{n}\|\nabla f\|_{n} .
$$


The condition that the left hand side of the previous inequality is finite defines the space $L(\infty, n)$, and, moreover, we have (cf. [11])

$$
L(\infty, n) \subset B W H .
$$

Since redefining the $L(p, q)$ spaces by means of replacing $f^{* *}$ by $f^{* *}-f^{*}$ gives equivalent norms when the parameters are in the usual range of the classical theory 5 , we now have a unified method to prove an inequality that includes the problematic case $p=n$,

$$
\int_{0}^{|\Omega|}\left(\left(f^{* *}(t)-f^{*}(t)\right) t^{1 / p-1 / n}\right)^{q} \frac{d t}{t} \leq c_{n} \int_{0}^{|\Omega|}\left(t^{1 / p}|\nabla f|^{* *}(t)\right)^{q} \frac{d t}{t} .
$$

In fact, let us note that we can do this even if the measure is infinite,

$$
\left\{\int_{0}^{\infty}\left(\left(f^{* *}(t)-f^{*}(t)\right)\right)^{n} \frac{d t}{t}\right\}^{1 / n} \leq c_{n}\||\nabla f|\|_{n}
$$

In this notation, the original Hardy-Littlewood-O'Neil program 6 has been completed7:

$$
\|f\|_{L\left(p_{n}, p\right)} \leq c\||\nabla f|\|_{L^{p}}, 1 \leq p \leq n .
$$

The improvement on Brezis-Wainger-Hansson is possible due to the fact that the class $L(\infty, n)$ is not a linear space.

A posteriori, we also understood that an inequality obtained by Kolyada on the unit cube [61, that is exactly like (4), except that the oscillation condition on the left hand side is given in terms of $f^{*}(t)-f^{*}(2 t)$, could also have been used for the Euclidean inequalities above (cf. [100]). Moreover, Tartar 111] (cf. also [69]) earlier than [11, but apparently after [10, had also obtained, using a different approach based on truncations, a discrete version of a result that, with some work, can be seen to be equivalent to (6). This was not clearly understood at the time we wrote 11 .

From the point of view of the development of our program, we draw the following from this section: Redefining the target spaces using oscillations, and using the pointwise inequality (4), we could treat all the cases of the classical Sobolev inequalities in an optimal unified manner.

To proceed further with our program of understanding more general geometries the question we faced next was: What would be a substitute for (4) when dealing with other geometries? Since the oscillation condition $f^{* *}(t)-f^{*}(t)$ is a general construct and, in fact, can be understood from an approximation point of view (cf. [56]), we concentrated our efforts on understanding better the right hand side of the inequality.

\footnotetext{
${ }^{5}$ For a detailed discussion on equivalences between different Lorentz ${ }^{*}$ norms* we refer to 11, [56], 72].

${ }^{6}$ The proof to deal with the case $p=1$ is slightly different and hinges on a variant of (4), namely (cf. [85])

$$
\int_{0}^{t}\left(f^{* *}(s)-f^{*}(s)\right) s^{1 / n} \frac{d s}{s} \leq c \int_{0}^{t}\left(|\nabla f|^{*}(s)\right) d s .
$$

${ }^{7}$ Actually (6) also makes sense, and gives sharp results, when $p>n$ (cf. [79] Chapter 9]).
} 


\section{Martin-Milman-Pustylnik meet Maz'ya}

The Gagliardo-Nirenberg inequality

$$
\|f\|_{L^{n^{\prime}}} \leq c\||\nabla f|\|_{L^{1}}, f \in C_{0}^{\infty}\left(\mathbb{R}^{n}\right),
$$

is well known to be equivalent to the isoperimetric inequality (cf. Maz'ya 87] and the references therein.) Moreover, using the chain rule and the scale properties of the $L^{p}$ spaces (here scale = Hölder's inequality) one can readily see that (7) implies "all" the classical Sobolev inequalities: simply consider positive $f$ and apply (7) to $f^{\alpha}$ for suitable $\alpha$ combined with the chain rule and Hölder's inequality (cf. [107]).

From our experience we knew that we could also derive "all the Sobolev inequalities" from the rearrangement inequality

$$
f^{* *}(t)-f^{*}(t) \leq c_{n} t^{1 / n}|\nabla f|^{* *}(t) .
$$

So it was natural to ask what was the connection between (7) and (8)? The key for us was the method of truncation that had been devised by Maz'ya (cf. 87]) and Talenti [110, combined with one natural idea that comes from interpolation theory, or more precisely, from the work of Marcinkiewicz and Alberto Calderón 24]. The idea simply put is to do the (smooth) cut-offs using the rearrangement of the function to be truncated evaluated at a fixed point $t$. This leads to pointwise rearrangement inequalities. In this way we could show that (7) is equivalent to (8)! (cf. [85])

One advantage of (8) is that since it is a pointwise inequality it gives all the results, even for non $L^{p}$ norms. This was another innovation from the work of Alberto Calderón 24: While previously to prove interpolation theorems one had to specify the spaces in advance this was not longer necessary in A. P. Calderón's theory. This was crucially important in our program since the norms than one needs to use to measure the integrability gains depend on the geometry. We should also point out that for Euclidean geometries pointwise rearrangement inequalities had been devised and applied by Talenti (cf. [110] and the references therein), and since then we have been applied by many authors working on non-linear PDEs...(cf. [41, 104)) In connection with compactness of Sobolev embeddings connected with the developments in this section we refer to [103], [85] and the many references therein.

\section{The Gaussian Inequality: Ledoux's inequality}

The next item on our agenda was a test case: The Gaussian world. The questions here were: How to formulate the basic inequality (8) in the Gaussian context? What type of symmetrization was needed to replace the symmetric rearrangement? What inequality would take the role of the Gagliardo-Nirenberg inequality in the Gaussian world? The answer to the last question had been already provided by Ledoux (cf. 65]). Ledoux's inequality is connected with 
the use of the Maz'ya isoperimetric profile, usually referred to as isoperimetric profile $I_{\gamma}$, associated with Gaussian measure in $\mathbb{R}^{n}$,

$$
I_{\gamma}(t)=\inf \left\{\gamma^{+}(A): \gamma(A)=t\right\},
$$

where $\gamma^{+}$is the Minkowski content associated with Gaussian measure defined for Borel sets $A$ by

$$
\gamma^{+}(A)=\lim _{h \rightarrow 0} \frac{\gamma\left(\left(A_{h}\right)\right)-\gamma(A)}{h},
$$

where $A_{h}=\{x: d(x, A)<h\}$, and $d$ is the usual $\mathbb{R}^{n}$ distance between $\{x\}$ and the set $A$. For Lip functions $f$, combining the isoperimetric inequality

$$
I_{\gamma}(\gamma(\{|f|>t\})) \leq \gamma^{+}(\{|f|>t\}),
$$

with the Gaussian co-area formuld9, we have

$$
\begin{aligned}
\int_{0}^{\infty} I_{\gamma}(\gamma(\{|f|>t\})) d t & \leq \int_{0}^{\infty} \gamma^{+}(\{|f|>t\}) d t \\
& =\||\nabla f|\|_{L^{1}\left(\mathbb{R}^{n}, \gamma\right)} .
\end{aligned}
$$

This is exactly the same mechanism one can use in the Euclidean world 10 . Now, in the Gaussian world, the isoperimetric profile $I_{\gamma}(t)$ has the following estimate (with constants independent of the dimension)

$$
I_{\gamma}(t) \simeq t\left(\log \frac{1}{t}\right)^{1 / 2}, t \in(0,1 / 2),
$$

while for the Euclidean isoperimetric profile we have

$$
I_{n}(t)=c_{n} t^{1-1 / n}, t>0 .
$$

Note that in the Euclidean case,

$$
\begin{aligned}
\int_{0}^{\infty} I_{n}(|\{|f|>t\}|) d t & =\int_{0}^{\infty} I_{n}\left(\lambda_{f}(t)\right) d t \\
& =\int_{0}^{\infty} I_{n}(t) d f^{*}(t) \\
& =\int_{0}^{\infty} f^{*}(t) d I_{n}(t) \\
& =c_{n} \frac{1}{n^{\prime}} \int_{0}^{\infty} f^{*}(t) t^{1 / n^{\prime}} \frac{d t}{t} .
\end{aligned}
$$

\footnotetext{
${ }^{8}$ The isoperimetric profile was introduced by Maz'ya in the sixties and further developed by him in a number of publications (cf. 87. and the references therein). Independently, this useful tool was developed in parallel by geometers (cf. [12] and the references therein) and probabilists (cf. 66] and the references therein), and as we shall see plays an important role in our work formulating Sobolev pointwise inequalities on rearrangements.

${ }^{9}$ In the general metric case it becomes the co-area inequality (cf. [18]).

${ }^{10}$ apparently this is true in the whole universe..
} 
In other words, the logarithmic Sobolev inequality 11 of Ledoux (9) is the ana$\log u e^{12}$ of the Gagliardo-Nirenberg in Gaussian world. Using the method of "symmetrization by truncation" we could then derive the Gaussian version of (8)

$$
f_{\gamma}^{* *}(t)-f_{\gamma}^{*}(t) \leq \frac{t}{I_{\gamma}(t)}|\nabla f|_{\gamma}^{* *}(t)
$$

The remarkable fact is that in the Euclidean world (i.e. taking rearrangements using Lebesgue measure and using the corresponding formula for the Euclidean isoperimetric profile) this inequality is exactly (8) ! In fact, these inequalities are equivalent to the corresponding isoperimetric inequalities in each of these geometries!

Also note that, the isoperimetric profile automatically selects the spaces that need to be involved! Our approach in 73 is based on these ideas. For example, in the Gaussian case, from (10) we obtain directly the following version of a logarithmic Sobolev inequality

$$
\int\left(f_{\gamma}^{* *}(t)-f_{\gamma}^{*}(t)\right)^{2}\left(\log \frac{1}{t}\right) d t \leq \int\left(|\nabla f|_{\gamma}^{* *}(t)\right)^{2} d t
$$

Moreover, since the Gaussian isoperimetric profile does not depend on the dimension 13 the inequalities in this case are dimension free! For a complete development we refer to 73 and through this paper we refer to many other important references. Some references connected with this section.. 6], 7], 8, [9] [13, [19, [21, [22], 25], 35], 36], 37], 47], 53], 64]...

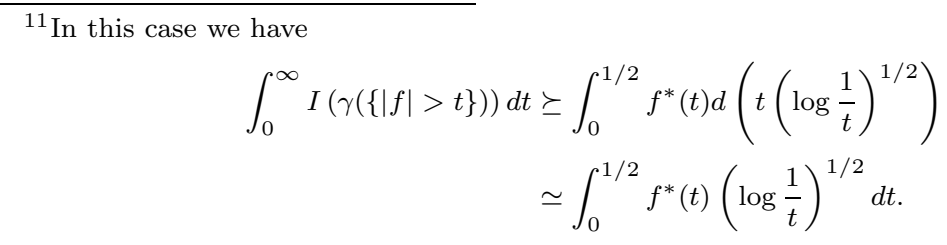

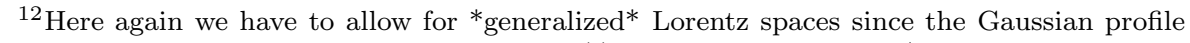
although concave is not increasing. Indeed, $I(t)$ is symmetric about $1 / 2$. Also note that the inequality

$$
\int_{0}^{\infty} I(\gamma(\{|f|>t\})) d t \leq\|\nabla f\|_{L^{1}\left(R^{n}, \gamma\right)}
$$

holds for functions $f$ that do not vanish at the boundary. For example, for $f=1$, the right hand is zero and

$$
\begin{aligned}
\int_{0}^{\infty} I(\gamma(\{|f|>t\})) d t & \left.=\int_{0}^{1} I(1)\right) d t \\
& =\int_{0}^{1} 0 d t \\
& =0 .
\end{aligned}
$$

\footnotetext{
${ }^{13}$ It is well known that the sets that realize the isoperimetric inequality are always hyperspaces: i.e. all but one of the variables are free.
} 


\section{The Metric Case}

When working on the Gaussian inequalities, we realized early on that, with a suitable definition of modulus of the gradient $14|\nabla f|$, and having at hand an associated co-area formula $\sqrt{15}$, we could indeed prove (10) in the general setting of metric measure spaces (this was informally first announced in 73 and more formally in [75). Fortunately, all the tools that we need to implement this insight had already been developed by Bobkov-Houdre [18.

In this generalized setting we work with connected metric probability spaces $(\Omega, \mu)$ The isoperimetric profile $I_{\mu}=I_{(\Omega, d, \mu)}$ is defined by

$$
I_{(\Omega, d, \mu)}(t)=\inf _{A}\left\{\mu^{+}(A): \mu(A)=t\right\},
$$

where $\mu^{+}(A)$ is the perimeter or Minkowski content of the Borel set $A \subset X$, defined by

$$
\mu^{+}(A)=\lim \inf _{h \rightarrow 0} \frac{\mu\left(A_{h}\right)-\mu(A)}{h},
$$

where $A_{h}=\{x \in \Omega: d(x, A)<h\}$. We assume that $I$ is continuous, concave, symmetric about $1 / 2$, and zero at zero. Further we assume 16 that for each $c \in \mathbb{R}$, and each $f \in \operatorname{Lip}(\Omega),|\nabla f(x)|=0, \mu-a . e$. on the set $\{x: f(x)=c\}$. The associated isoperimetric inequality can be formulated as: for all Borel sets A

$$
I(\mu(A)) \leq \mu^{+}(A)
$$

\subsection{The Gagliardo-Nirenberg-Bobkov-Houdre inequality}

We have the following result due to Bobkov-Houdre (cf. [18]),

$\int_{0}^{\infty} I_{\mu}\left(\mu_{f}(t)\right) d t \leq\||\nabla f|\|_{L^{1}(\Omega)}$, for all $f \in \operatorname{Lip}(\Omega) \Leftrightarrow$ isoperimetric inequality.

The reason the isoperimetric inequality is necessary is that given any Borel set $A$ we can find a sequence of Lip functions $\left\{f_{n}\right\}_{n}$ such that $\left\|\left|\nabla f_{n}\right|\right\|_{L^{1}(\Omega)} \rightarrow \mu^{+}(A)$, while $\int_{0}^{\infty} I_{\mu}\left(\mu_{f_{n}}(t)\right) d t \rightarrow I_{\mu}(\mu(A))$ (cf. [18]).

\footnotetext{
${ }^{14}$ For all Lipschitz function $f$ on $\Omega$, the modulus of the gradient is defined by

$$
|\nabla f(x)|=\limsup _{d(x, y) \rightarrow 0} \frac{|f(x)-f(y)|}{d(x, y)} .
$$

${ }^{15}$ The strong connection between the co-area formula and Sobolev embeddings had already been emphasized by Maz'ya in his pionnering fundamental work in the early sixties (cf. 87]).

${ }^{16}$ See [52. Using an approximation argument developed by E. Milman [91, Remark 3.3] it is possible to prove the main inequalities of this paper without this assumption (cf. 78] and the forthcoming [84]).
} 


\subsection{Generalized Pólya-Szegö}

One big difference between the general probability metric case and the Gaussian case is the lack of symmetry. In particular, in the Gaussian world $\left(\mathbb{R}^{n}, \gamma_{n}\right)$ there is a natural choice for a distinguished rearrangement that replaces the symmetric rearrangement. Let

$$
\phi_{n}(x)=(2 \pi)^{-n / 2} e^{-\frac{|x|^{2}}{2}}, x \in \mathbb{R}^{n}, \Phi(r)=\int_{-\infty}^{r} \phi_{1}(t) d t, \mathbb{R} \in \mathbb{R},
$$

then the Gaussian profile is given by (cf. Borell [22] and Sudakov-Tsirelson [109])

$$
I_{\gamma}(t)=\phi_{1}\left(\Phi^{-1}(t)\right), t \in[0,1] .
$$

The classical Euclidean spherical decreasing rearrangement is replaced by

$$
f_{\gamma_{n}}^{\circ}(x)=f_{\gamma_{n}}^{*}\left(\Phi\left(x_{1}\right)\right),
$$

and we have the Erhard analogue of Pólya-Szegö (cf. [35, [37, 773])

$$
\left.\mid \nabla\left(f_{\gamma_{n}}^{\circ}\right)\right)\left.\right|_{\gamma} ^{* *}(t) \leq|\nabla f|_{\gamma}^{* *}(t) .
$$

In general there is no apparent symmetry and thus no distinguished rearrangement. This led us to formulate the following alternative inequality which extends (13) to the probability metric case the Pólya-Szegö inequality in the Euclidean geometry and the Erhard inequality in the Gaussian case: for all $f \in \operatorname{Lip}$, we have (cf. [77])

$$
\int_{0}^{t}\left(I_{\mu}(\cdot) \frac{d}{d t}\left(-f_{\mu}^{*}(\cdot)\right)\right)^{*}(s) d s \leq \int_{0}^{t}|\nabla f|_{\mu}^{*}(s) d s,
$$

where the rearrangement inside the integral on the left hand side is with respect to Lebesgue measure. The usual formulation of Pólya-Szegö as a norm inequality follows directly from (14) and the Calderón-Hardy-Littlewood principle. To see this result in detail let us recall that a rearrangement invariant space $X(\Omega, \mu)$, has a representation $\bar{X}(0,1)$ such that

$$
\|f\|_{X(\Omega)}=\left\|f_{\mu}^{*}\right\|_{X(0,1)} .
$$

Now, since $|\nabla f|_{\mu}^{*}=\left(|\nabla f|_{\mu}^{*}\right)^{*}$, we see that if (14) holds then by the CalderónHardy-Littlewood principle,

$$
\left\|I_{\mu}(\cdot) \frac{d}{d t}\left(-f_{\mu}^{*}(\cdot)\right)\right\|_{X(0,1)} \leq\left\||\nabla f|_{\mu}^{*}\right\|_{X(0,1)}=\left\||\nabla f|_{\mu}^{*}\right\|_{X(\Omega)},
$$

which by abuse of notation (since no confusion can arise) we write as

$$
\left\|I_{\mu}(t) \frac{d}{d t}\left(-f_{\mu}^{*}(t)\right)\right\|_{X} \leq\||\nabla f|\|_{X}
$$


The proof of these inequalities follows by smooth truncation. Indeed, if we apply (12) to the smooth truncations,

$$
f_{t_{1}}^{t_{2}}(x)=\left\{\begin{array}{cc}
t_{2}-t_{1} & \text { if }|f(x)| \geq t_{2} \\
|f(x)|-t_{1} & \text { if } t_{1}<|f(x)| \leq t_{2} \\
0 & \text { if }|f(x)| \leq t_{1}
\end{array}\right.
$$

and we use the fact that

$$
\int_{t_{1}}^{t_{2}} I_{\mu}\left(\mu_{f}(s)\right) d s=\int_{0}^{t_{2}-t_{1}} I_{\mu}\left(\mu_{f_{1}}^{t_{2}}(s)\right) d s
$$

then we find that

$$
\int_{t_{1}}^{t_{2}} I_{\mu}\left(\mu_{f}(s)\right) d s \leq \int_{\left\{t_{1}<|f|<t_{2}\right\}}|\nabla f| d \mu .
$$

A carefu 17 "change of variables argument $s \mapsto f_{\mu}^{*}(u)$ " (cf. [77]) on the left hand side gives

$$
\int_{t_{1}}^{t_{2}} I_{\mu}(u) \frac{d}{d t}\left(-f_{\mu}^{*}\right)(u) d u=\int_{f_{\mu}^{*}\left(t_{2}\right)}^{f_{\mu}^{*}\left(t_{1}\right)} I_{\mu}\left(\mu_{f}(s)\right) d s \leq \int_{\left\{f_{\mu}^{*}\left(t_{2}\right)<|f|<f_{\mu}^{*}\left(t_{1}\right)\right\}}|\nabla f| d \mu .
$$

Thus, for any set $E=\bigcup\left(a_{i}, b_{i}\right)$ union of disjoint intervals, with $|E|=t$, we have

$$
\begin{aligned}
& \int_{E} I(u) \frac{d}{d t}\left(-f_{\mu}^{*}\right)(u) d u \leq \sum_{i} \int_{\left\{f_{\mu}^{*}\left(b_{i}\right)<|f|<f_{\mu}^{*}\left(a_{i}\right)\right\}}|\nabla f| d \mu
\end{aligned}
$$

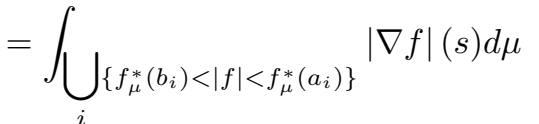

$$
\begin{aligned}
& \leq \int_{0}^{\sum \mu\left\{f_{\mu}^{*}\left(b_{i}\right)<|f|<f_{\mu}^{*}\left(a_{i}\right)\right\}}|\nabla f|_{\mu}^{*}(s) d \mu \\
& \leq \int_{0}^{\sum\left(b_{i}-a_{i}\right)}|\nabla f|_{\mu}^{*}(s) d \mu \\
& =\int_{0}^{t}|\nabla f|_{\mu}^{*}(s) d \mu \text {. }
\end{aligned}
$$

By approximation,

$$
\begin{aligned}
\int_{0}^{t}\left(I(\cdot) \frac{d}{d t}\left(-f^{*}\right)(\cdot)\right)^{*}(u) d u & =\sup _{|E|=t} \int_{E} I(u) \frac{d}{d t}\left(-f^{*}\right)(u) d u \\
& \leq \int_{0}^{t}|\nabla f|_{\mu}^{*}(s) d \mu
\end{aligned}
$$

and (14) follows.

\footnotetext{
${ }^{17}$ Under the assumption that $f$ is $L i p$ the calculations below can be justified (cf. [74] [77, 68])
} 


\subsection{Maz'ya-Talenti inequality}

Likewise, with a similar direct proof, we can extend a well known inequality apparently obtained first by Maz'ya [88] and independently, but later, by Talenti (cf. [85] and the references therein). In our setting this inequality takes the following form (cf. [77)

$$
I_{\mu}(t) \frac{d}{d t}\left(-f_{\mu}^{*}(t)\right) \leq \frac{d}{d t} \int_{\left\{|f|>f^{*}(t)\right\}}|\nabla(f)|_{\mu}^{*}(s) d s .
$$

In fact, it is also easy to see that the argument given above shows that (15) implies (14). Indeed, starting with (15), and considering first sets which are unions of disjoint intervals, we arrive at

$$
\sup _{|E|=t} \int_{E} I_{\mu}(u) \frac{d}{d t}\left(-f_{\mu}^{*}\right)(u) d u \leq \int_{0}^{t}|\nabla f|_{\mu}^{*}(s) d \mu .
$$

\subsection{Basic rearrangement inequality}

Next we observe that the previous arguments with intervals also show (cf. [77]) that, integrating by parts, we obtain (recall that under our assumptions $f_{\mu}^{*}$ is absolutely continuous (cf. [68)

$$
f_{\mu}^{* *}(t)-f_{\mu}^{*}(t)=\frac{1}{t} \int_{0}^{t} s \frac{d}{d t}\left(-f_{\mu}^{*}\right)(s) d s .
$$

Consequently,

$$
f_{\mu}^{* *}(t)-f_{\mu}^{*}(t) \leq \frac{1}{t} \frac{t}{I_{\mu}(t)} \int_{0}^{t} I_{\mu}(s) \frac{d}{d t}\left(-f_{\mu}^{*}\right)(s) d s \quad \text { (since } \frac{s}{I_{\mu}(s)} \text { is increasing). }
$$

By now, we have discussed several inequalities that can be used to control $\int_{0}^{t} I_{\mu}(s)\left(-f_{\mu}^{*}(s)\right)^{\prime} d s$. For example, using the definition of rearrangement and the generalized Pólya-Szegö inequality (14), we have

$$
\begin{aligned}
\int_{0}^{t} I_{\mu}(s) \frac{d}{d t}\left(-f_{\mu}^{*}\right)(s) d s & \leq \int_{0}^{t}\left(I_{\mu}(\cdot) \frac{d}{d t}\left(-f_{\mu}^{*}\right)(\cdot)\right)^{*}(s) d s \\
& \leq \int_{0}^{t}|\nabla f|_{\mu}^{*}(s) d s .
\end{aligned}
$$

Consequently, combining the last inequality with (16) we arrive to the familiar

$$
\begin{aligned}
f_{\mu}^{* *}(t)-f_{\mu}^{*}(t) & \leq \frac{1}{I_{\mu}(t)} \int_{0}^{t}|\nabla f|_{\mu}^{*}(s) d s \\
& =\frac{t}{I(t)}|\nabla f|_{\mu}^{* *}(t) .
\end{aligned}
$$

Using a standard approximation argument (like the one outlined above) (cf. [77, [78) we see that (17) implies the isoperimetric inequality (11).

Let us also remark that is fairly easy to give a direct proof of (17) (cf. Section 8 below) 


\subsection{Self Improvement}

Under stronger assumptions on the isoperimetric profile we can get stronger inequalities. For example, suppose that

$$
\int_{t}^{1} \frac{I_{\mu}(s)}{s} \frac{d s}{s} \leq c \frac{I_{\mu}(t)}{t}, t \in(0,1) .
$$

Then, for all $f \in \operatorname{Lip}(\Omega)$,

$$
\int_{0}^{t}\left(f_{\mu}^{* *}(s)-f_{\mu}^{*}(s)\right) \frac{I(s)}{s} d s \leq \int_{0}^{t}\left(\frac{I(\cdot)}{(\cdot)}\left[f_{\mu}^{* *}(\cdot)-f_{\mu}^{*}(\cdot)\right]\right)^{*} d s \leq C \int_{0}^{t}|\nabla f|_{\mu}^{*}(s) d s .
$$

This extends the sharp form of the Gagliardo-Nirenberg inequality to metric spaces that have "Euclidean like profiles". Indeed, suppose that $\frac{I(s)}{s} \simeq s^{1 / n^{\prime}}$, then for $f \in \operatorname{Lip}_{0}(\Omega)$, we have

$$
\int_{0}^{t}\left(f_{\mu}^{* *}(s)-f_{\mu}^{*}(s)\right) s^{1 / n^{\prime}} \frac{d s}{s} \leq c_{n} \int_{0}^{t}|\nabla f|_{\mu}^{*}(s) d s .
$$

Consequently, letting $t \rightarrow \infty$,

$$
\int_{0}^{\infty}\left(f_{\mu}^{* *}(s)-f_{\mu}^{*}(s)\right) s^{1 / n^{\prime}} \frac{d s}{s} \leq c_{n}\|\nabla f\|_{1} .
$$

\subsection{Poincaré inequalities and generalized Pólya-Szegö}

Once again we follow [77. To discuss Poincaré inequalities in the metric setting we introduced the Hardy isoperimetric operators (cf. [77]). Let us define

$$
Q_{I}(f)(t)=\chi_{(0,1 / 2)}(t) \int_{t}^{1 / 2} f(s) \frac{d s}{I_{\mu}(s)} .
$$

Suppose that for positive functions supported on $(0,1 / 2)$

$$
\left\|Q_{I}(f)\right\|_{X} \leq\left\|Q_{I}\right\|_{X \rightarrow X}\|f\|_{X}
$$

Then, the following Poincaré inequality holds

$$
\left\|g-\int_{\Omega} g\right\|_{X} \preceq\left\|Q_{I}\right\|_{X \rightarrow X}\||\nabla g|\|_{X} .
$$

Indeed, for $g \in \operatorname{Lip}(\Omega), t \in(0,1 / 2)$ we can write,

$$
\begin{aligned}
g_{\mu}^{*}(t)-g_{\mu}^{*}(1 / 2) & =\int_{t}^{1 / 2}\left(-g_{\mu}^{*}(s)\right)^{\prime} d s \\
& =\int_{t}^{1 / 2} I_{\mu}(s)\left(-g_{\mu}^{*}(s)\right)^{\prime} \frac{d s}{I_{\mu}(s)} \\
& =Q_{I}\left(I_{\mu}(.)\left(-g_{\mu}^{*}(.)\right)^{\prime}\right) .
\end{aligned}
$$


Thus,

$$
g_{\mu}^{*}(t)=Q_{I}\left(I_{\mu}(.)\left(-g_{\mu}^{*}(.)\right)^{\prime}\right)+g_{\mu}^{*}(1 / 2)
$$

Now,

$$
\left\|g_{\mu}^{*}\right\|_{X} \leq\left\|g_{\mu}^{*} \chi_{(0,1 / 2)}\right\|_{X}+\left\|g_{\mu}^{*} \chi_{(1 / 2,1)}\right\|_{X}
$$

and

$$
\begin{aligned}
\left\|g_{\mu}^{*} \chi_{(1 / 2,1)}\right\|_{X} & \leq g_{\mu}^{*}(1 / 2)\left\|\chi_{(1 / 2,1)}\right\|_{X} \\
& =g_{\mu}^{*}(1 / 2)\left\|\chi_{(0,1 / 2)}\right\|_{X} \\
& \leq\left\|g_{\mu}^{*} \chi_{(0,1 / 2)}\right\|_{X},
\end{aligned}
$$

yields

$$
\left\|g_{\mu}^{*}\right\|_{X} \preceq\left\|g_{\mu}^{*} \chi_{(0,1 / 2)}\right\|_{X}
$$

which combined with (20) gives

$$
\begin{aligned}
\left\|g_{\mu}^{*}\right\|_{X} & \preceq\left\|g_{\mu}^{*} \chi_{(0,1 / 2)}\right\|_{X} \\
& \leq \| Q_{I}\left(I_{\mu}(\cdot) \frac{d}{d t}\left(-g_{\mu}^{*}\right)(\cdot)\left\|_{X}+\right\| g_{\mu}^{*}(1 / 2) \|_{X}\right. \\
& \leq\left\|Q_{I}\right\|_{X \rightarrow X}\left\|\left(I_{\mu}(\cdot) \frac{d}{d t}\left(-g_{\mu}^{*}\right)\right)\right\|_{X}+\left\|g_{\mu}^{*}(1 / 2)\right\|_{X} \\
& \leq\left\|Q_{I}\right\|_{X \rightarrow X}\|\mid \nabla g\|\left\|_{X}+g_{\mu}^{*}(1 / 2)\right\| 1 \|_{X} .
\end{aligned}
$$

Now, by Chebyshev's inequality $g_{\mu}^{*}(1 / 2) \leq 2\|g\|_{1}$. Therefore,

$$
\begin{aligned}
\|g\|_{X} & =\left\|g_{\mu}^{*}\right\|_{X} \\
& \preceq\left\|Q_{I}\right\|_{X \rightarrow X}\left(\||\nabla g|\|_{X}+\|g\|_{1}\right) .
\end{aligned}
$$

Applying the previous inequality to $g-\int_{\Omega} g$,

$$
\left\|g-\int_{\Omega} g\right\|_{X} \preceq\left\|Q_{I}\right\|_{X \rightarrow X}\left(\||\nabla g|\|_{X}+\left\|g-\int_{\Omega} g\right\|_{1}\right),
$$

and combining with Cheeger's inequality yields

$$
\begin{aligned}
\left\|g-\int_{\Omega} g\right\|_{1} & \preceq\|\nabla g\|_{1} \\
& \leq\|\nabla g\|_{X} .
\end{aligned}
$$

Thus we finally arrive at

$$
\left\|g-\int_{\Omega} g\right\|_{X} \preceq\left\|Q_{I}\right\|_{X \rightarrow X}\||\nabla g|\|_{X} .
$$




\subsection{Identity associated with Poincaré}

For the sake of completeness we note that the previous discussion also shows that for $g \in \operatorname{Lip}(\Omega)$ we have (cf. 19)

$$
g_{\mu}^{*}(t)-g_{\mu}^{*}(1 / 2)=Q_{I}\left(I_{\mu}(\cdot) \frac{d}{d t}\left(-g_{\mu}^{*}\right)(\cdot)\right)
$$

This section is based entirely on [77] but builds on a long list of important contributors we refer to [77] for a more complete list of references. For versions of Pólya-Szegö in the classical format (norm inequality) we refer to the books 60, 68] as well as Talenti [110, Almgren-Lieb [3], and the references therein. We should also mention the work of the French geometer Gallot [42] on symmetrization inequalities on manifolds, which as we discovered a posteriori, is close in spirit to our development here. The important paper by D. Bakry, T. Coulhon, M. Ledoux and L. Saloff-Coste [5] opened our eyes early on to the possibilities afforded by the masterful use of cut-offs.

\section{Coulhon Inequalities}

The previous section dealt with Sobolev inequalities in probability metric spaces. The next natural questions are: how to deal with more general metric measure spaces? Secondly: what is the rearrangement version of $L^{p}$ Sobolev inequalities for $p>1$ ? In this situation we need to replace the isoperimetric profile by a suitable capacitary profile and the formulation of the inequalities is somewhat more complicated (cf. [78]). However, one can take a different tack (cf. [80]) and work with an equivalent formulation of the Sobolev inequalities due to Coulhon (cf. [28, [27, 26]) and Bakry-Coulhon-Ledoux [5].

Let $(\Omega, d, \mu)$ be a metric measure space, let $p \in[1, \infty]$, and let $\phi$ be an increasing function on the positive half line. We consider Coulhon inequalities of the form

$$
\left(S_{\phi}^{p}\right) \quad\|f\|_{p} \leq \phi\left(\|f\|_{0}\right)\||\nabla f|\|_{p}, f \in \operatorname{Lip}_{0}(\Omega),
$$

where $\|f\|_{0}=\mu\{$ support $(f)\}$.

An important point to keep in mind is that the function $\phi$ must then be connected to the geometry; but now we do not specify what this connection is in advance! In [78] we find rearrangement inequalities that characterize $\left(S_{\phi}^{p}\right)$. As was to be expected the rearrangement inequalities incorporate in their formulation the function $\phi$.

To see the connection with the previous discussions let us consider the case $p=1$. We shall show that this case is connected with the isoperimetric inequality. Before doing so, let us mention that our approach in this section is independent and, indeed, can be seen as an alternative route to rearrangement inequalities discussed in previous sections. Let us then consider the connection between $\left(S_{\phi}^{1}\right)$ and a rearrangement inequality of the form

$$
f_{\mu}^{* *}(t)-f_{\mu}^{*}(t) \leq \phi(t)|\nabla f|_{\mu}^{* *}(t), f \in \operatorname{Lip}_{0}(\Omega) .
$$


Suppose (21) holds where $\phi$ is a given increasing continuous function. Let $t>0$; multiplying both sides of (21) by $t$, we obtain

$$
t\left(f_{\mu}^{* *}(t)-f_{\mu}^{*}(t)\right) \leq \phi(t) \int_{0}^{t}|\nabla f|_{\mu}^{*}(s) d s .
$$

Since formally $f_{\mu}^{*}(t)=\mu_{f}^{-1}(t)$, drawing a diagram it is easy to see that

$$
\begin{aligned}
t\left(f_{\mu}^{* *}(t)-f_{\mu}^{*}(t)\right) & =\int_{0}^{t} f_{\mu}^{*}(s) d s-t f_{\mu}^{*}(t) \\
& =\int_{f^{*}(t)}^{\infty} \mu_{f}(s) d s .
\end{aligned}
$$

Consequently, if we let $t=\|f\|_{0}$, we see that $f_{\mu}^{*}\left(\|f\|_{0}\right)=0, \int_{f_{\mu}^{*}\left(\|f\|_{0}\right)}^{\infty} \mu_{f}(s) d s=$ $\|f\|_{1}$, and $\int_{0}^{\|f\|_{0}}|\nabla f|_{\mu}^{*}(s) d s=\||\nabla f|\|_{1}$. Thus,

$$
\|f\|_{1} \leq \phi\left(\|f\|_{0}\right)\||\nabla f|\|_{1} .
$$

Suppose on the other hand that an $\left(S_{\tilde{\phi}}^{1}\right)$ condition holds. For $f \in \operatorname{Lip}_{0}(\Omega)$, and for $t>0$, let us apply the $\left(S_{\phi}^{1}\right)$ condition to $\left[f-f_{\mu}^{*}(t)\right]_{+}$. We compute,

$$
\left\|\left[f-f_{\mu}^{*}(t)\right]_{+}\right\|_{0}=\mu\left\{f>f_{\mu}^{*}(t)\right\} \leq t,
$$

moreover, since $\int_{\left\{f=f^{*}(t)\right\}}\left|\nabla\left[f(x)-f_{\mu}^{*}(t)\right]\right| d x=0$, we have

$$
\left\|\nabla\left[f-f_{\mu}^{*}(t)\right]_{+}\right\|_{L^{1}}=\int_{\left\{f>f^{*}(t)\right\}}|\nabla f|_{\mu}^{*}(s) d s .
$$

We also have

$$
\begin{aligned}
\left\|\left[f-f_{\mu}^{*}(t)\right]_{+}\right\|_{1} & =\int_{\left\{f>f^{*}(t)\right\}}\left[f(s)-f_{\mu}^{*}(t)\right]_{+} d \mu(s) \\
& =\int_{0}^{\infty}\left[f_{\mu}^{*}(x)-f_{\mu}^{*}(t)\right]_{+} d x \\
& =\int_{0}^{t}\left(f_{\mu}^{*}(x)-f_{\mu}^{*}(t)\right) d x \\
& =t\left(f_{\mu}^{* *}(t)-f_{\mu}^{*}(t)\right) .
\end{aligned}
$$

Inserting these calculations in (22), we find

$$
t\left(f_{\mu}^{* *}(t)-f_{\mu}^{*}(t)\right) \leq t \phi(t)\left(\frac{1}{t} \int_{0}^{t}|\nabla f|_{\mu}^{*}(s) d s\right) .
$$

In other words, we have shown that

$$
\left(S_{\phi}^{1}\right) \Leftrightarrow\left(f_{\mu}^{* *}(t)-f_{\mu}^{*}(t)\right) \leq \phi(t)|\nabla f|_{\mu}^{* *}(t), f \in \operatorname{Lip}_{0}(\Omega) .
$$


In particular, if $I_{\mu}(t)$ is concave then $\phi(t)=\frac{t}{I_{\mu}(t)}$ is increasing, and we have

$$
\left(S_{\frac{t}{I_{\mu}(t)}}^{1}\right) \Leftrightarrow\left(f_{\mu}^{* *}(t)-f_{\mu}^{*}(t)\right) \leq \frac{t}{I_{\mu}(t)}|\nabla f|_{\mu}^{* *}(t), f \in \operatorname{Lip}_{0}(\Omega) .
$$

Let us now show that the function $\frac{t}{I_{\mu}(t)}$ is optimal. Suppose that an $\left(S_{\phi}^{1}\right)$ condition holds, then it is easy to see, by approximation, that $\frac{t}{I_{\mu}(t)} \leq \phi(t)$ (cf. [80]). The fact that $\left(S_{\frac{t}{I_{\mu}(t)}}^{1}\right)$ itself holds is a direct consequence of the co-area inequality for Lip functions on metric spaces (cf. [18]). It is instructive to see the details. Suppose that $f \in \operatorname{Lip}_{0}(\Omega)$, then from $I_{\mu}(\mu(A)) \leq \mu^{+}(A)$, for all Borel sets, we have the Bobkov-Houdre inequality

$$
\int_{0}^{\infty} I_{\mu}\left(\mu_{f}(t)\right) d t \leq\||\nabla f|\|_{1} .
$$

Now, since $\frac{I_{\mu}(t)}{t}$ decreases, and we obviously have $\mu_{f}(t) \leq\|f\|_{0}$, we see that

$$
\begin{aligned}
\int_{0}^{\infty} I_{\mu}\left(\mu_{f}(t)\right) d t & =\int_{0}^{\infty} \mu_{f}(t) \frac{I_{\mu}\left(\mu_{f}(t)\right)}{\mu_{f}(t)} d t \\
& \geq \int_{0}^{\infty} \mu_{f}(t) d t \frac{I_{\mu}\left(\|f\|_{0}\right)}{\|f\|_{0}} .
\end{aligned}
$$

Combining these inequalities, we see that for the choice of $\phi(t)=\frac{t}{I_{\mu}(t)}$ we do indeed have

$$
\|f\|_{1}=\int_{0}^{\infty} \mu_{f}(t) d t \leq \phi\left(\|f\|_{0}\right)\||\nabla f|\|_{1} .
$$

For example, in the Euclidean space $\mathbb{R}^{n}, I(t)=d_{n} t^{1-1 / n}, \phi(t) \simeq t^{1 / n}$ and the best possible $\left(S_{\phi}^{1}\right)$ inequality can be written as

$$
\|f\|_{1} \leq c_{n}\|f\|_{0}^{1 / n}\||\nabla f|\|_{1} .
$$

With the choice of $\phi(t)=t^{1 / n}$, and $p=2$, Coulhon [28] shows the equivalence of the $S_{\phi}^{2}$ inequality with the classical Nash inequality

$$
\|f\|_{2}^{1+2 / n} \leq c_{n}\|f\|_{1}^{2 / n}\||\nabla f|\|_{2} .
$$

The general characterization of Coulhon inequalities in terms of rearrangements is given by the following (cf. [80])

Theorem 3 Let $(\Omega, d, \mu)$ be a connected Borel metric measure space as described above, and let $p \in[1, \infty)$. The following statements are equivalent

1. $\left(S_{\phi}^{p}\right)$ holds, i.e.

$$
\|f\|_{p} \leq \phi\left(\|f\|_{0}\right)\||\nabla f|\|_{p}, \text { for all } f \in \operatorname{Lip}_{0}(\Omega) .
$$


2. Let $k \in \mathbb{N}$ be such that $k<p \leq k+1$, then for all $f \in \operatorname{Lip}_{0}(\Omega)$

$$
\left(\frac{f_{(p)}^{* *}(t)}{\phi_{(p)}(t)}\right)^{1 / p}-\left(\frac{f_{(p)}^{*}(t)}{\phi_{(p)}(t)}\right)^{1 / p} \leq 2^{\frac{k+1}{p}-1}\left(|\nabla f|_{(p)}^{* *}(t)\right)^{1 / p}
$$

where

$$
f_{(p)}^{*}(t)=\left(f^{*}(t)\right)^{p}, f_{(p)}^{* *}(t)=\frac{1}{t} \int_{0}^{t} f_{(p)}^{*}(s) d s, \phi_{(p)}(t)=(\phi(t))^{p} .
$$

We note that for $p=1$ the inequality (23) of Theorem 3 coincides with (17). This new characterization for $p \geq 1$ is independent of [77, and, in fact, the proof we gave above provides a new approach to (17) as well.

For the details of the proof of Theorem 3 we must refer to 80 .

We cannot resist to make a connection between Coulhon's inequalities and the theory of factorization of operators. Local operators (cf. 102, 2, 295 and the references therein) satisfy conditions of the form

$$
\|T f\|_{Y} \leq \phi\left(\|f\|_{0}\right)\|f\|_{X} .
$$

Self-improvements in this setting are expressed via factorization and change of density. We think that the factorization of Sobolev inequalities could be an interesting line of investigation to pursue .

\section{Connection with the work of Emanuel Milman}

In this section, following [77, we consider a connection with the work of Emanuel Milman'18 (cf. 89, 90, 91, 92, 93, 94)).

For metric measure spaces $(\Omega, d, \mu)$ obtained from a $C^{\infty}$ complete oriented $n$-dimensional Riemannian manifold $(M, g)$, where $d$ is the induced geodesic distance and $\mu$ is absolutely continuous with respect to $\mathrm{dvol}_{M}$, it is known that the corresponding isoperimetric profile satisfies (cf. [12]) that $I_{(\Omega, d, \mu)}(t)$ is continuous, $I_{(\Omega, d, \mu)}(t)>0$ for $t \in(0,1)$, and moreover,

$$
I_{(\Omega, d, \mu)}(t)=I_{(\Omega, d, \mu)}(1-t), \forall t \in[0,1] .
$$

E. Milman further assumes some convexity conditions: $d \mu=e^{-\Psi} d v o l_{M}$, where $\Psi$ is such that $\Psi \in C^{2}(M)$, and as tensor fields $\operatorname{Ric}_{g}+\operatorname{Hess}_{g}(\Psi) \geq 0$ on $M$. In this case it then follows that $I_{(\Omega, d, \mu)}$ is also concave (cf. [89] and the extensive list of references therein). Under such conditions, E. Milman shows the equivalence of Cheeger's inequality, Poincaré's inequality and concentration inequalities! More precisely, using a variety of different tools, including the semigroup approach of Ledoux, E. Milman has shown that (cf. also Ledoux's 67. streamlined approach to E. Milman's results in 92]).

\footnotetext{
${ }^{18}$ We refer to E. Milman's papers for an account of the history of the problem. Emanuel, who belongs to the Milman family of mathematicians that includes David (grandfather), Vitali (father), Pierre (uncle) (cf. [97]), is no direct relation to Mario Milman.
} 
Theorem 4 (E. Milman) Let $(\Omega, d, \mu)$ be a metric probability space satisfying E. Milman's convexity conditions. Then following statements are equivalent (E1) Cheeger's inequality: there exists a positive constant $C$ such that

$$
I_{(\Omega, d, \mu)} \geq C t, \quad t \in(0,1 / 2] .
$$

(E2) Poincaré's inequality: there exists a positive constant $P$ such that for all $f \in \operatorname{Lip}(\Omega)$,

$$
\left\|f-m_{e}\right\|_{L^{2}(\Omega)} \leq P\||\nabla f|\|_{L^{2}(\Omega)} .
$$

(E3) Exponential concentration: there exist positive constants $c_{1}, c_{2}$ such that for all $f \in \operatorname{Lip}(\Omega)$ with $\|f\|_{L i p(\Omega)} \leq 1$,

$$
\mu\left\{\left|f-m_{e}\right|>t\right\} \leq c_{1} e^{-c_{2} t}, \quad t \in(0,1) .
$$

(E4) First moment inequality: there exists a positive constant $F$ such that for all $f \in \operatorname{Lip}(\Omega)$ with $\|f\|_{\operatorname{Lip}(\Omega)} \leq 1$,

$$
\left\|f-m_{e}\right\|_{L^{1}(\Omega)} \leq F .
$$

Remark 5 For the optimal relationship between the concentration profile and Cheeger's constant under suitable convexity conditions see [94].

\subsection{Isoperimetric Hardy type}

We single out a class of metric probability spaces that are suitable for our analysis (cf. [77, [76]).

Definition 6 We shall say that a probability metric space $(\Omega, d, \mu)$ is of isoperimetric Hardy type if for any given isoperimetric estimator $I$, the following are equivalent for all r.i. spaces $X=X(\Omega), Y=Y(\Omega)$.

1. There exists a constant $c=c(X, Y)$ such that for all $f \in \operatorname{Lip}(\Omega)$

$$
\left\|f-\int_{\Omega} f d \mu\right\|_{Y} \leq c\||\nabla f|\|_{X} .
$$

2. There exists a constant $c_{1}=c_{1}(X, Y)>0$ such that for all positive functions $f \in \bar{X}$, with $\operatorname{supp}(f) \subset(0,1 / 2)$ we have

$$
\left\|Q_{I} f\right\|_{\bar{Y}} \leq c_{1}\|f\|_{\bar{X}},
$$

where $Q_{I}$ is the isoperimetric Hardy operator

$$
Q_{I} f(t)=\chi_{(0,1 / 2)}(t) \int_{t}^{1 / 2} f(s) \frac{d s}{I(s)} .
$$

For spaces of isoperimetric type it is possible to give a very simple proof of the E. Milman's equivalences (cf. [77]). 
Theorem 7 Suppose that $(\Omega, d, \mu)$ is a metric probability space of isoperimetric Hardy type. Then

$$
(E 1) \Leftrightarrow(E 2) \Leftrightarrow(E 3) \Leftrightarrow(E 4) .
$$

Example 8 All the model spaces studied in [77] (including Gaussian space (cf. [73]) are of Hardy isoperimetric type.

For further results connecting our work with E. Milman's work we refer to [77] and [78. For example, the following result of E. Milman can be understood in the context of Hardy isoperimetric type (cf. [77).

Theorem 9 Let $(\Omega, d, \mu)$ be a space satisfying E. Milman's convexity conditions. Let $1 \leq q<\infty$, and let $N$ be a Young's function such that $\frac{N(t)^{1 / q}}{t}$ is nondecreasing, and there exists $\alpha>\max \left\{\frac{1}{q}-\frac{1}{2}, 0\right\}$ such that $\frac{N\left(t^{\alpha}\right)}{t}$ non-increasing. Then, the following statements are equivalent:

(E5) $\left(L_{N}, L^{q}\right)$ Poincaré inequality holds: there exists a positive constant $P$ such that for all $f \in \operatorname{Lip}(\Omega)$

$$
\left\|f-m_{e}\right\|_{L_{N}(\Omega)} \leq P\||\nabla f|\|_{L^{q}(\Omega)} .
$$

(E6) Any isoperimetric profile estimator I satisfies: there exists a constant c $>0$ such that $I(t) \geq c \frac{t^{1-1 / q}}{N^{-1}(1 / t)}, \quad t \in(0,1 / 2]$.

E. Milman's work can also be seen as providing a program to unify Sobolev inequalities in different geometries. For more on this we refer to our paper [78] where, generalizing E. Milman's work, we in particular show why Lorentz spaces appear as optimal target spaces for Sobolev embeddings.

\section{Transference and dimensionless inequalities}

In this section we follow [77 and 81 to show how our pointwise inequalities can be used to transfer Sobolev inequalities from one geometry to another. The transference is of special interest when it is implemented to replace Sobolev inequalities that carry dimensional constants by weaker, but dimensionless, Sobolev inequalities. Inequalities independent of the dimension play an increasingly important role in approximation theory and its applications (cf. 46]). To see how our pointwise inequalities are relevant for this task let us recall that our typical Sobolev inequality on a metric probability space $(\Omega, d, \mu)$, takes the form

$$
\left\|\left(f_{\mu}^{* *}(t)-f_{\mu}^{*}(t)\right) \frac{I_{\mu}(t)}{t}\right\|_{\bar{X}} \leq c\left\||\nabla f|_{\mu}^{* *}\right\|_{\bar{X}} .
$$

Now suppose that the metric probability space $(\Omega, d, \mu)$ is of "Gaussian isoperimetric type", that is suppose that for some universal constant independent of the dimension, it holds

$$
I_{(\Omega, \mu)}(t) \succeq t\left(\log \frac{1}{t}\right)^{\frac{1}{2}}, \text { on }\left(0, \frac{1}{2}\right) ;
$$


then we can obviously replace $\frac{I_{\mu}(t)}{t}$ by $\left(\log \frac{1}{t}\right)^{\frac{1}{2}}$ in (24), and in this fashion *transfer* the Gaussian inequality to $(\Omega, d, \mu)$ :

$$
\left\|\left(f_{\mu}^{* *}(t)-f_{\mu}^{*}(t)\right)\left(\log \frac{1}{t}\right)^{\frac{1}{2}}\right\|_{\bar{X}} \leq c\left\||\nabla f|_{\mu}^{* *}\right\|_{\bar{X}} .
$$

This argument shows how the Gaussian log Sobolev inequalities can be transferred to $(\Omega, d, \mu)$ with constants independent of the dimension. In particular, since $Q_{n}$, the open unit cube in $\mathbb{R}^{n}$, is of Gaussian type with constant equal to 1 (cf. [105), the Gaussian Sobolev inequalities can be transferred to $Q_{n}$, with constants independent of the dimension.

This answered a question of Triebel [113 (cf. 77, 62, 63, 81, 114]) and the references therein). For example, the non-homogeneous form of these results take the following form

$$
\|f\|_{L^{q}(\log L)^{q / 2}\left(Q_{n}\right)} \leq C(q)\left(\||\nabla f|\|_{L^{q}\left(Q_{n}\right)}+\|f\|_{L^{q}\left(Q_{n}\right)}\right), f \in C_{0}^{\infty}\left(Q_{n}\right) .
$$

In turn, this result was recently improved by Krbec-Fiorenza-Schmeisser [40], using the spaces $L_{\left(q, q^{\prime}\right.}\left(Q_{n}\right)$, originally introduced by Iwaniec-Sbordone-Fiorenza (cf. [54, [38]), and characterized by Fiorenza-Karadzhov [39], using extrapolation (cf. [59]) as follows

$$
\|f\|_{L_{\left(q, q^{\prime}\right.}\left(Q_{n}\right)} \simeq \int_{0}^{1}\left(\int_{0}^{t} f^{*}(s)^{q} d s\right)^{1 / q} \frac{d t}{t\left(\log \frac{1}{t}\right)^{\frac{1}{2}}} .
$$

The (non homogeneous) result in [40] yields

$$
\|f\|_{L_{\left(q, q^{\prime}\right.}\left(Q_{n}\right)} \leq C(q)\left(\|\nabla f\|_{L^{q}\left(Q_{n}\right)}+\|f\|_{L^{q}\left(Q_{n}\right)}\right), f \in C_{0}^{\infty}\left(Q_{n}\right) .
$$

Consequently, since

$$
L_{\left(q, q^{\prime}\right.}\left(Q_{n}\right) \subset L^{q}(\log L)^{q / 2}\left(Q_{n}\right),
$$

this result provides an improvement upon (26).

In [81] we showed that the inequality (27) is connected with a different transference principle. We start by reformulating (25) as

$$
\left\|\left(f_{\mu}^{* *}(t)-f_{\mu}^{*}(t)\right) \chi_{(0,1 / 2)}(t) G_{\infty}(t)\right\|_{\bar{X}} \leq c\left\|G_{\infty}(t) \frac{t}{I(t)}\right\|_{L^{\infty}\left(0, \frac{1}{2}\right)}\left\||\nabla f|_{\mu}^{* *}\right\|_{\bar{X}},
$$

then (25) corresponds to the choice $G_{\infty}(t)=\left(\log \frac{1}{t}\right)^{\frac{1}{2}}$. Now, using the fact that $\frac{I_{\mu}(t)}{t}$ decreases we see that left hand side of (24) can be minorized as follows,

$$
\begin{aligned}
\left\|\left(f_{\mu}^{* *}(\cdot)-f_{\mu}^{*}(\cdot)\right) \frac{I_{\mu}(\cdot)}{(\cdot)}\right\|_{\bar{X}} & \geq\left\|\left(f_{\mu}^{* *}(\cdot)-f_{\mu}^{*}(\cdot)\right) \chi_{(0, t)}(\cdot) \frac{I_{\mu}(\cdot)}{(\cdot)}\right\|_{\bar{X}} \\
& \geq\left\|\left(f_{\mu}^{* *}(\cdot)-f_{\mu}^{*}(\cdot)\right) \chi_{(0, t)}(\cdot)\right\|_{\bar{X}} \frac{I_{\mu}(t)}{t} .
\end{aligned}
$$


Therefore, we have

$$
\left\|\left(f_{\mu}^{* *}(\cdot)-f_{\mu}^{*}(\cdot)\right) \chi_{(0, t)}(\cdot)\right\|_{\bar{X}} \leq c \frac{t}{I_{\mu}(t)}\left\||\nabla f|_{\mu}^{* *}\right\|_{\bar{X}} .
$$

Now, if $G_{1}$ is such that $\left(\int_{0}^{1} G_{1}(t) \frac{t}{I(t)} d t\right)<\infty$, it follows immediately from (28) that

$$
\int_{0}^{1}\left\|\left(f_{\mu}^{* *}(\cdot)-f_{\mu}^{*}(\cdot)\right) \chi_{(0, t)}(\cdot)\right\|_{\bar{X}} G(t) d t \leq C\left(\int_{0}^{1} G_{1}(t) \frac{t}{I(t)} d t\right)\left\||\nabla f|_{\mu}^{* *}\right\|_{\bar{X}} .
$$

For example, let $G_{1}(t)=\frac{1}{t\left(\log \frac{1}{t}\right)^{\frac{1}{2}}}$, and suppose the following (stronger) Gaussian isoperimetric transference condition is satisfied,

$$
\int_{0}^{1} \frac{d t}{I(t)\left(\log \frac{1}{t}\right)^{\frac{1}{2}}}<\infty
$$

Then, we have (cf. [81])

$$
\begin{aligned}
\left\|\left(f_{\mu}^{* *}(\cdot)-f_{\mu}^{*}(\cdot)\right)\left(\log \left(\frac{1}{\cdot}\right)\right)^{1 / 2}\right\|_{\bar{X}} & \leq c \int_{0}^{1}\left\|\left(f_{\mu}^{* *}(s)-f_{\mu}^{*}(s)\right) \chi_{(0, t)}(s)\right\|_{\bar{X}} \frac{d t}{t\left(\log \frac{1}{t}\right)^{\frac{1}{2}}} \\
& \leq c\left(\int_{0}^{1} \frac{d t}{I(t)\left(\log \frac{1}{t}\right)^{\frac{1}{2}}}\right)\left\||\nabla f|^{*}\right\|_{\bar{X}} \cdot
\end{aligned}
$$

Let us show a concrete application. Let

$$
I_{n}(t)=n\left(\gamma_{n}\right)^{1 / n} t^{1-1 / n}
$$

where $\gamma_{n}=\frac{\pi^{n / 2}}{\Gamma(1+n / 2)}$ is the measure of the unit ball in $\mathbb{R}^{n}$ (i.e. $I_{n}(t)$ is the isoperimetric profile associated to $\mathbb{R}^{n}$ ), and consider the function

$$
G_{1}(t)=\frac{1}{t \sqrt{\ln \left(\frac{1}{t}\right)}}, t \in(0,1) .
$$


Then,

$$
\begin{aligned}
\int_{0}^{1} \frac{t}{t I_{n}(t)} G_{1}(t) d t & =\frac{1}{n\left(\gamma_{n}\right)^{1 / n}} \int_{0}^{1} t^{1 / n} \frac{d t}{t\left(\ln \frac{1}{t}\right)^{\frac{1}{2}}} \\
& =\frac{1}{n\left(\gamma_{n}\right)^{1 / n}} \int_{0}^{\infty} z^{-\frac{1}{2}} e^{-z / n} d z \\
& =\frac{\sqrt{\pi} n^{\frac{1}{2}}}{n\left(\gamma_{n}\right)^{1 / n}} \\
& =\frac{\Gamma\left(1+\frac{n}{2}\right)^{1 / n}}{n^{\frac{1}{2}}} . \\
& =\left(\frac{n}{2}\right)^{1 / n} \frac{\Gamma\left(\frac{n}{2}\right)^{1 / n}}{n^{\frac{1}{2}}} \\
& \leq \frac{1}{\sqrt{2}}\left(\frac{n}{2}\right)^{1 / n} \\
& \leq c .
\end{aligned}
$$

Thus,

$$
\sup _{n} \int_{0}^{1} \frac{d t}{I_{n}(t)\left(\log \frac{1}{t}\right)^{\frac{1}{2}}}<\infty .
$$

As a consequence the following dimensionless Sobolev inequality holds,

$$
\int_{0}^{1}\left\|\left(f^{* *}(\cdot)-f^{*}(t)\right) \chi_{[0, t)}(\cdot)\right\|_{\bar{X}} \frac{d t}{t\left(\log \frac{1}{t}\right)^{1 / 2}} \leq C\left\||\nabla f|^{* *}\right\|_{\bar{X}} \cdot
$$

For $X=L^{q}$ and for $\Omega=Q_{n}$ this gives the result of [40].

\section{Rearrangement inequalities of Garsia-Rodemich type and Morrey's theorem}

To complement the results of previous sections we now consider fractional inequalities and the Morrey-Sobolev embedding theorem. Here we follow [79] where the reader will find a complete treatment together with many applications.

Let us describe model results that influenced our development in these directions.

Example 10 For all $f \in X\left(\mathbb{R}^{n}\right)+\dot{W}_{X}^{1}\left(\mathbb{R}^{n}\right)$, we have (cf. [70])

$$
f^{* *}(t)-f^{*}(t) \leq c_{n} \frac{\omega_{X}\left(t^{1 / n}, f\right)}{\phi_{X}(t)}, t>0,
$$

where $X=X\left(\mathbb{R}^{n}\right)$ is a rearrangement invariant space on $\mathbb{R}^{n}, \phi_{X}(t)=\left\|\chi_{A}\right\|_{X}$, with $|A|=t$, is the fundamental function of $X$, and $\omega_{X}$ be the modulus of 
continuity associated with $X$ :

$$
\omega_{X}(t, g)=\sup _{|h| \leq t}\|g(\cdot+h)-g(\cdot)\|_{X}, \text { for } g \in X .
$$

The inequality (30) can be formulated and proved on different levels of generality on the spaces, the domains; and indeed have a long history: We refer to [61], [57], [15], [70] and the references therein.

The associated questions to Example 10 are: What is the corresponding Gaussian result? More generally: What is the metric version? What is the role of dimension? What is the connection with isoperimetry? Even to formulate metric results we need to develop suitable tools. For example: What is an appropriate replacement for the modulus of continuity?

We shall need the following definition: Consider a connected, measure metric spaces $(\Omega, d, \mu)$ equipped with a finite Borel measure $\mu$. For measurable functions $u: \Omega \rightarrow \mathbb{R}$, the signed decreasing rearrangement of $u$, which we denote by $u_{\mu}^{s}$, is the right-continuous non-increasing function from $[0, \mu(\Omega))$ into $\mathbb{R}$ that is equimeasurable with $u$; i.e. $u_{\mu}^{s}$ satisfies

$$
\mu\{x \in \Omega: u(x)>t\}=m\left(\left\{z \in[0, \mu(\Omega)): u_{\mu}^{s}(z)>t\right\}\right), t \in \mathbb{R}
$$

The maximal average of $u_{\mu}^{s s}$ is defined by

$$
u_{\mu}^{s s}(t)=\frac{1}{t} \int_{0}^{t} u_{\mu}^{s}(z) d z,(t>0) .
$$

Example 11 In closely related work Garsia and his collaborators (cf. [45], [44], [43] and the references therein) obtained related inequalities. For example, in [44] and [43] for functions defined on the unit n-cube, and using signed rearrangements with respect the Lebesgue measure

$$
\left.\begin{array}{c}
f^{s}(x)-f^{s}(1 / 2) \\
f^{s}(1 / 2)-f^{s}(1-x)
\end{array}\right\} \leq c \int_{x}^{1} \frac{\omega_{L^{p}}\left(t^{1 / n}, f\right)}{t^{1 / p}} \frac{d t}{t}, x \in\left(0, \frac{1}{2}\right],
$$

where $\omega_{L^{p}}(t, f)$ the $L^{p}$ modulus of continuity. The extension from dimension 1 to dimension $n$ in these works was done through highly non trivial combinatorial inequalities (cf. 43]).

In this context we can ask similar questions to those posed in Example 10 ,

Example 12 In the work of Garsia and his collaborators one also finds another interesting use of rearrangement inequalities to extract continuity. Through a change of scale argument, inequalities on rearrangements were used, for example, to prove versions of Morrey's Sobolev theorem. For example, in the one dimensional case (cf. [44], [45], [43]) we have

$$
|f(x)-f(y)| \leq 2 c \int_{0}^{|x-y|} \frac{\omega_{L^{p}}(t, f)}{t^{1 / p}} \frac{d t}{t} ; x, y \in[0,1] .
$$


We now adapt the change of scale argument of Garsia et. al. combined with the rearrangement inequality (17) in the context of the unit cube to prove a version of Morrey's theorem. First, let us stipulate (cf. [79]) that one can rewrite the (17) in terms of signed rearrangements (i.e. we rearrange $f$ rather than its absolute value). Suppose that $p>n$, and let $f \in W_{1}^{1}(0,1)^{n}$. Starting with the fundamental theorem of calculus

$$
f^{s s}(0)-f^{s s}(1)=\int_{0}^{1}\left(f^{s s}(t)-f^{s}(t)\right) \frac{d t}{t}
$$

and the corresponding version of (17)

$$
f^{s s}(t)-f^{s}(t) \leq c_{n} \frac{t}{\min (t, 1-t)^{1-1 / n}}|\nabla f|^{* *}(t), \quad 0<t<1,
$$

we see that

$$
\begin{aligned}
f^{s s}(0)-f^{s s}(1) & \leq c_{n} \int_{0}^{1}|\nabla f|^{* *}(t) \frac{d t}{\min (t, 1-t)^{1-1 / n}} \\
& \leq c_{n, p}\||\nabla f|\|_{L^{p}}\left\|\frac{1}{\min (t, 1-t)^{1-1 / n}}\right\|_{L^{p^{\prime}(0,1)}} \text { (by Hölder's inequality) } \\
& =C_{n . p}\||\nabla f|\|_{L^{p}} .
\end{aligned}
$$

This works of course because for $p>n,\left\|\frac{1}{\min (t, 1-t)^{1-1 / n}}\right\|_{L^{p^{\prime}}}<\infty$. Now (recall we are working with signed rearrangements) since $f^{s s}(0)=e s s \sup _{x \in(0,1)^{n}} f$, and $f^{s s}(1)=\int_{0}^{1} f$, it thus follows that

$$
\text { ess } \sup _{x \in(0,1)^{n}} f-\int_{0}^{1} f=f^{s s}(0)-f^{s s}(1) \leq C_{n . p}\||\nabla f|\|_{L^{p}} .
$$

Applying (32) now to $-f$ yields

$$
\int_{0}^{1} f-e s s \inf _{x \in(0,1)^{n}} f \leq C_{n . p}\||\nabla f|\|_{L^{p}} .
$$

Therefore, adding (32) and (33) we obtain

$$
O s c\left(f ;(0,1)^{n}\right):=e s s \sup _{x \in(0,1)^{n}} f-e s s \inf _{x \in(0,1)^{n}} f \leq 2 C_{n . p}\||\nabla f|\|_{L^{p}} .
$$

Now, we scale: Apply the previous argument to the restriction of $f$ to a subcube $Q, f \chi_{Q}$. We obtain,

$$
O s c(f ; Q) \leq c_{n, p}\left\|\frac{t}{\min (t,|Q|-t)^{1-1 / n}}\right\|_{L^{p^{\prime}(0,|Q|)}}\||\nabla f|\|_{L^{p}(Q)} .
$$

By computation, it is now easy to see that we will have

$$
|f(y)-f(z)| \leq c_{n, p}|y-z|^{\left(1-\frac{n}{p}\right)}\||\nabla f|\|_{p} \text {, a.e. } y, z .
$$


To carry out our program in metric measure spaces $(\Omega, d, \mu)$ we first need to find a replacement for the modulus of continuity. In 79 we use the Peetre 19 $K$-functional defined by

$$
\begin{gathered}
K\left(t, f ; X(\Omega), S_{X}(\Omega)\right):= \\
\inf \left\{\|f-g\|_{X(\Omega)}+t\||\nabla g|\|_{X(\Omega)}: g \in S_{X}(\Omega)\right\},
\end{gathered}
$$

where for a rearrangement invariant space $X(\Omega), S_{X}(\Omega)=\{f \in \operatorname{Lip}(\Omega)$ : $\left.\||\nabla f|\|_{X(\Omega)}<\infty\right\}$. In the classical setting we have (cf. [15], [16])

$$
\begin{aligned}
K\left(t, f ; X\left(\mathbb{R}^{n}\right), \dot{W}_{X}^{1}\left(\mathbb{R}^{n}\right)\right) & :=\inf \left\{\|f-g\|_{X}+t\||\nabla g|\| \|_{X}: g \in \dot{W}_{X}^{1}\left(\mathbb{R}^{n}\right)\right\} \\
& \simeq \omega_{X}(t, f) .
\end{aligned}
$$

We can thus think of $K\left(t, f ; X(\Omega), S_{X}(\Omega)\right)$ as generalized "modulus of continuity", and we have (cf. [79] and also [86])

Theorem 13 (cf. [79]) Let $(\Omega, d, \mu)$ be a metric measure space that satisfies our standard assumptions. Then,

(i) For all rearrangement invariant spaces $X(\Omega)$, and for all $f \in X(\Omega)+$ $S_{X}(\Omega)$,

$$
\begin{gathered}
f_{\mu}^{s s}(t)-f_{\mu}^{s}(t) \leq 16 \frac{K\left(\frac{t}{I_{\Omega}(t)}, f ; X(\Omega), S_{X}(\Omega)\right)}{\phi_{X}(t)}, t \in(0, \mu(\Omega) / 2), \\
\left(f-f_{\Omega}\right)_{\mu}^{* *}(t)-\left(f-f_{\Omega}\right)_{\mu}^{*}(t) \leq 16 \frac{K\left(\frac{t}{I_{\Omega}(t)}, f ; X(\Omega), S_{X}(\Omega)\right)}{\phi_{X}(t)}, t \in(0, \mu(\Omega)),
\end{gathered}
$$

where

$$
f_{\Omega}=\frac{1}{\mu(\Omega)} \int_{\Omega} f d \mu
$$

(ii) Conversely, suppose that $G:(0, \mu(\Omega)) \rightarrow \mathbb{R}_{+}$, is a continuous function, which is concave and symmetric around $\mu(\Omega) / 2$, and that there exists a constant $c>0$ such that for all $f \in X(\Omega)+S_{X}(\Omega)$,

$$
f_{\mu}^{s s}(t)-f_{\mu}^{s}(t) \leq c \frac{K\left(\frac{t}{G(t)}, f ; X(\Omega), S_{X}(\Omega)\right)}{t}, t \in(0, \mu(\Omega) / 2) .
$$

Then, there exists a constant $c>0$ such that for all $t \in(0, \mu(\Omega))$,

$$
G(t) \leq c I_{\Omega}(t)
$$

\footnotetext{
${ }^{19}$ Independently, and in parallel, A. Calderón and his student Oklander defined and studied the $K$-functional, and real interpolation, e.g. in Oklander's thesis at the University of Chicago (cf. 98]).

${ }^{20}$ In other words we assume that (34) holds for $X=L^{1}(\Omega)$, and with $\frac{t}{G(t)}$ replacing $\frac{t}{I_{\Omega}(t)}$.
} 
Following the analysis of [79, Chapter 10], one can show that for a metric probability space $(\Omega, d, \mu)$, that satisfies our standard assumptions, the Garsia inequality (31) takes the following form,

$$
\left.\begin{array}{c}
f^{s}(x)-f^{s}(1 / 2) \\
f^{s}(1 / 2)-f^{s}(1-x)
\end{array}\right\} \leq c \int_{x}^{1} \frac{K\left(\frac{t}{I_{\Omega}(t)}, f ; X(\Omega), S_{X}(\Omega)\right)}{\phi_{X}(t)} \frac{d t}{t}, x \in\left(0, \frac{1}{2}\right] .
$$

For the scaling argument we outlined above we need an extra assumption. We say that an isoperimetric inequality relative to $G$ holds, if there exists a positive constant $C_{G}$ such that

$$
I_{G}(s) \geq C_{G} \min \left(I_{\Omega}(s), I_{\Omega}(\mu(G)-s)\right) .
$$

We say that the metric measure space $(\Omega, d, \mu)$ has the uniform relative isoperimetric property, if there exist $C>0, \delta>0$, such that for any $x \in \Omega$, and any open ball $B_{\alpha}(x)$ centered on $x$, with $\mu\left(B_{\alpha}(x)\right)=\alpha(0<\alpha<\delta)$, the relative isoperimetric profile $I_{B_{\alpha}(x)}$ satisfies:

$$
I_{B_{\alpha}(x)}(s) \geq C \min \left(I_{\Omega}(s), I_{\Omega}(\alpha-s)\right), \quad 0<s<\alpha .
$$

Then we have (cf. [79, Chapter 4])

Theorem 14 Let $(\Omega, d, \mu)$ be a metric measure space that satisfies our standard assumptions and, moreover, has the relative uniform isoperimetric property. Let $X$ be a r.i. space in $\Omega$ such that

$$
\left\|\frac{1}{I_{\Omega}(s)}\right\|_{\bar{X}^{\prime}}<\infty
$$

Then, if $f \in X+S_{X}(\Omega)$ satisfies

$$
\int_{0}^{\mu(\Omega)} \frac{K\left(\phi_{X}(t)\left\|\frac{1}{I_{\Omega}(s)} \chi_{(0, t)}(s)\right\|_{\bar{X}^{\prime}}, f ; X, S_{X}(\Omega)\right)}{\phi_{X}(t)} \frac{d t}{t}<\infty,
$$

it follows that $f$ is essentially bounded and essentially continuous.

For applications we need to have explicit computations of the relevant $K$-functional. We refer to [31, 32, 33] for a treatment of $K$-functionals in one dimensional Gaussian measure. For other relevant computations of $K$-functionals see the appendix of [79].

For further developments we must refer to [79]. In connection with this section we should also mention [115] and the references therein.

\section{Higher Order Inequalities}

In this section we work with Euclidean domains $\Omega$ and measure spaces of the form $d \mu(x)=w(x) d x$, and we formulate higher order pointwise inequalities by 
iteration 21 that extend our previous work in 71 and $[96$. The basic inequality here reads as follows. We assume that $\int d \mu(x)<\infty$, and we let $I_{\mu}:=I$, then for sufficiently smooth $f$ we have that for $k \geq 2$, (cf. [82]))

$$
\begin{aligned}
f^{* *}(t)-f^{*}(t) & \leq \frac{1}{(k-1) !} \frac{t}{I(t)} \int_{t}^{1 / 2}\left|d^{k} f\right|^{* *}(u)\left(\frac{1}{I(u)}\left(\int_{t}^{u} \frac{d z}{I(z)}\right)^{k-1}\right) d u \\
& +\frac{1}{(k-1) !} \sum_{j=1}^{k-1}\left(\frac{t}{I(t)}\left(\int_{t}^{1 / 2} \frac{d z}{I(z)}\right)^{k-j-1}\right)\left\|\left|d^{k-j} f\right|\right\|_{1}, 0<t<1 / 2 .
\end{aligned}
$$

As a corollary we obtain,

$f^{* *}(t) \leq c_{k} \int_{t}^{1 / 2}\left|d^{k} f\right|^{* *}(s) \frac{1}{I(s)}\left(\int_{t}^{s} \frac{d z}{I(z)}\right)^{k} d s+\sum_{j=1}^{k} c_{j}(t)\left\|\left|d^{k-j} f\right|\right\|_{1}, 0<t<1 / 2$

where $c_{j}(t)=\left(\int_{t}^{1 / 2} \frac{d z}{I(z)}\right)^{k-j}$.

In particular, one can easily show that this result implies recent iterated inequalities that appeared in [108].

Theorem 15 Let $X, Y$ r.i spaces. Suppose that the operator $T$ defined by

$$
T f(t)=\int_{t}^{1 / 2} \frac{f(u)}{I(u)}\left(\int_{t}^{u} \frac{d z}{I(z)}\right)^{k-1} d u
$$

is bounded from $X$ to $Y$. Then

$$
\|u\|_{Y} \leq c\left(\left\|\left|d^{k} f\right|^{* *}\right\|_{X}+\sum_{j=1}^{k}\left\|\left|d^{k-j} f\right|\right\|_{1}\right) .
$$

Acknowledgement $\mathbf{1 6}$ We are grateful to E. Milman for a number of useful comments that helped improve the presentation.

\section{References}

[1] D. R. Adams and L. I. Hedberg, Function spaces and potential theory, Grund. Math. 314, Springer-Verlag, 1999.

[2] G. D. Allen, Locally Continuous Operators II, Indiana Univ. Math. J. 38 (1989), 711-743.

\footnotetext{
${ }^{21}$ The iteration of Sobolev inequalities is not a new idea (cf. 87, Corollary 6.9/1 in page 379 and Theorem 7.6.5 in page 430.]), the novelty here, if any, is the iteration of pointwise rearrangement inequalities
} 
[3] F. Almgren and E. Lieb, Symmetric Decreasing Rearrangement is sometimes continuous, J. Amer. Math. Soc. 2 (1989), 683-773.

[4] A. Alvino, G. Trombetti and P. L. Lions, On optimization problems with prescribed rearrangements, Nonlinear Anal. 13 (1989), 185-220.

[5] D. Bakry, T. Coulhon, M. Ledoux and L. Saloff-Coste, Sobolev inequalities in disguise, Indiana Univ. Math. J. 44 (1995) 1033-1074.

[6] F. Barthe, Levels of concentration between exponential and Gaussian, Ann. Fac. Sci. Toulouse Math. 10 (2001), 393-404.

[7] F. Barthe, Log-concave and spherical models in isoperimetry, Geom. Funct. Anal. 12 (2002), 32-55.

[8] F. Barthe, P. Cattiaux and C. Roberto, Isoperimetry between exponential and Gaussian, Orlicz hyper-contractivity and isoperimetry, Rev. Mat. Iber. 22 (2006), 993-1067.

[9] F. Barthe, P. Cattiaux and C. Roberto, Isoperimetry between exponential and Gaussian, Electronic Journal of Probability 12 (2007), 1212-1237.

[10] J. Bastero, M. Milman and F. Ruiz, On the connection between weighted norm inequalities, commutators and real interpolation, preprint, Sem A. Galdeano, 1996.

[11] J. Bastero, M. Milman and F. Ruiz, A note on $L(\infty, q)$ spaces and Sobolev embeddings, Indiana Mathematics Journal 52 (2003), 1215-1230.

[12] V. Bayle, Propriétés de concavité du profil isopérimétrique et applications. Ph.D. thesis, Institut Joseph Fourier, Grenoble (2004).

[13] W. Beckner and M. Persson, On sharp Sobolev embedding and the logarithmic Sobolev inequality, Bull. London Math. Soc. 30 (1998), 80-84

[14] C. Bennett, R. DeVore, and R. Sharpley, Weak- $L^{\infty}$ and BMO, Annals of Math. 113 (1981), 601-611.

[15] C. Bennett and R. Sharpley, Interpolation of Operators, Academic Press, Boston, 1988.

[16] J. Bergh and J. Löfström, Interpolation spaces. An introduction, SpringerVerlag, Berlin-Heidelberg-New York, 1976.

[17] S. G. Bobkov. Isoperimetric and analytic inequalities for log-concave probability measures, Ann. Probab. 27 (1999), 1903-1921.

[18] S.G. Bobkov and C. Houdré, Some connections between isoperimetric and Sobolev-type inequalities, Mem. Amer. Math. Soc. 129 (1997).

[19] S. G. Bobkov and B. Zegarlinski, Entropy bounds and isoperimetry, Mem. Amer. Math. Soc. 176 (2005). 
[20] S. G. Bobkov and B. Zegarlinski, Distributions with slow tails and ergodicity of Markov semigroups in infinite dimensions, in A. Laptev (ed), Around the research of Vladimir Maz'ya I: Function Spaces, Springer, 2010, pp 13-79.

[21] C. Borell, The Ehrhard inequality, C. R. Math. Acad. Sci. Paris 337 (2003), 663-666.

[22] C. Borell, The Brunn-Minkowski inequality in Gauss space, Invent. Math. 30 (1975), 207-216.

[23] H. Brezis and S. Wainger, A note on limiting cases of Sobolev embeddings and convolution inequalities, Comm. Partial Diff. Eq. 5 (1980), 773-789.

[24] A. P. Calderón, Spaces between $L^{1}$ and $L^{\infty}$ and the theorem of Marcinkiewicz, Studia Math. 26 (1966), 273-299.

[25] A. Cianchi and L. Pick, Optimal Gaussian Sobolev embeddings, J. Funct. Anal. 256 (2009), 3588-3642.

[26] T. Coulhon, Espaces de Lipschitz et inégalités de Poincaré, J. Funct. Anal. 136 (1996), 81-113.

[27] T. Coulhon, Dimensions at infinity for Riemannian manifolds, Pot. Anal. 4 (1995), 335-344.

[28] T. Coulhon, Heat kernel and isoperimetry on non-compact Riemmanian manifolds, Contemporary Mathematics 338 (2003), 65-99.

[29] M. Cwikel, B. Jawerth and M. Milman, A note on extrapolation of inequalities, preprint, 2010.

[30] M. Cwikel and E. Pustylnik, Sobolev type embeddings in the limiting case, J. Fourier Anal. Appl. 4 (1998), 433-446.

[31] Z. Ditzian and V. Totik, Moduli of Smoothness, Springer-Verlag, New York, 1987.

[32] Z. Ditzian and K. G. Ivanov, Strong converse inequalities, J. D'Analise Math. 61 (1993), 61-111.

[33] Z. Ditzian and D. S. Lubinsky, Jackson and Smoothness theorems for Freud weights in $L_{p}(0<p<\infty)$, Constr. Approx. 13 (1997), 99-152.

[34] D. E. Edmunds and W. D. Evans, Hardy operators, function spaces and embeddings, Springer-Verlag, Berlin, 2004.

[35] A. Ehrhard, Symétrisation dans l'espace de Gauss, Math. Scand. 53 (1983), 281-301. 
[36] A. Ehrhard, Sur l'inégalité de Sobolev logarithmique de Gross, Séminaire de Probabilités XVI I I, Lecture Notes in Math. 1059, 194-196, SpringerVerlag, 1984.

[37] A. Ehrhard, Inégalités isopérimétriques et intégrales de Dirichlet gaussiennes, Ann. Scient. Ec. Norm. Sup. 17 (1984), 317-332.

[38] A. Fiorenza, Duality and reflexivity in grand Lebesgue spaces, Collect. Math. 51, (2000), 131-148.

[39] A. Fiorenza and G. E. Karadzhov, Grand and Small Lebesgue Spaces and their analogs, Z. Anal. Anwendungen, 23 (2004), 657-681.

[40] A. Fiorenza, M. Krbec and H. J. Schmeisser, An improvement of dimension-free Sobolev imbeddings in r.i. spaces, preprint.

[41] L. Fontana and C. Morpurgo, Optimal limiting embeddings for $\Delta$-reduced Sobolev spaces in $L^{1}$, Ann. de l'Inst. Henri Poincaré (C) Non Linear Analysis, to appear.

[42] S. Gallot, Inégalités isopérimétriques et analytiques sur les variétés Riemanniennes, Astérisque No. 163-164 (1988), 31-91.

[43] A. M. Garsia, Combinatorial inequalities and smoothness of functions, Bull. Amer. Math. Soc. 82 (1976), 157-170.

[44] A. M. Garsia, A remarkable inequality and the uniform convergence of Fourier series, Indiana Univ. Math. J. 25 (1976), 85-102.

[45] A. Garsia and E. Rodemich, Monotonicity of certain functionals under rearrangement, Ann. Inst. Fourier 24 (1974), 67-116.

[46] M. Griebel, Sparse grids and related approximation schemes for higher dimensional problems, in L. Pardo, A. Pinkus, E. Suli, and M. Todd, editors, Foundations of Computational Mathematics (FoCM05), Santander, pp 106-161, Cambridge University Press, 2006.

[47] L. Gross, Logarithmic Sobolev inequalities, Amer. J. Math. 97 (1975), 1061-1083.

[48] P. Hajlasz, Sobolev inequalities, truncation method, and John domains, Papers in Analysis, Rep. Univ. Jyväskylä Dep. Math. Stat. 83, Univ. Jyväskylä, Jyväskylä, 2001, pp 109-126.

[49] P. Hajlasz and P. Koskela, Sobolev met Poincaré, Mem. Amer. Math. Soc. 145 (2000), 101 pages.

[50] K. Hansson, Imbedding theorems of Sobolev type in potential theory, Math Scand 45 (1979), 77-102. 
[51] L. I. Hedberg, On Maz'ya's work in potential theory and the theory of function spaces, The Maz'ya anniversary collection, Vol. 1 (Rostock, 1998), 7-16, Oper. Theory Adv. Appl. 109, Birkhäuser, Basel, 1999.

[52] J. Heinonen, Lectures on Analysis on metric spaces, Lecture Notes, 1996, University of Michigan.

[53] C. Houdre, M. Ledoux, E. Milman and M. Milman, Concentration, functional inequalities and isoperimetry, Contemporary Mathematics 545, 2011.

[54] T. Iwaniec and C. Sbordone, On the integrability of the Jacobian under minimal hypotheses, Arch. Rational Mech. Anal. 119 (1992), 129-143.

[55] B. Jawerth and M. Milman, Extrapolation theory with applications, Mem. Amer. Math. Soc. 89 (1991), no. 440.

[56] B. Jawerth and M. Milman, Interpolation of Weak Type Spaces, Math. Z. 201 (1989), $509-520)$

[57] H. Johnen and K. Scherer, On the equivalence of the K-functional and moduli of continuity and some applications, in Constructive theory of functions of several variables, Lecture Notes in Math. 571, pp. 119-140, Springer, Berlin, 1977.

[58] G. E. Karadzhov and Q. Mehmood, Optimal Regularity Properties of the Generalized Sobolev Spaces, J. Funct. Spaces Appl. (2013).

[59] G. E. Karadzhov and M. Milman, Extrapolation Theory: New Results and Applications, J. Approx. Th. 133 (2005), 38-99.

[60] S. Kesavan, Symmetrization and applications, World Scientific, 2006.

[61] V.I. Kolyada, Rearrangements of functions and embedding theorems, Uspekhi Mat. Nauk 44 (1989) 61-95; transl. in: Russian Math. Surveys 44 (1989), 73-117.

[62] M. Krbec and H. J. Schmeisser, On dimension-free Sobolev imbeddings I, J. Math. Anal. Appl. 387 (2012), 114-125.

[63] M. Krbec and H. J. Schmeisser, On dimension-free Sobolev imbeddings II, Rev. Mat. Complutense 25 (2012), 247-265.

[64] M. Ledoux, Isoperimetry and Gaussian Analysis, Ecole d'Eté de Probabilités de Saint-Flour 1994, Springer Lecture Notes 1648, pp 165-294, Springer-Verlag, 1996.

[65] M. Ledoux, Isopérimétrie et inégalitées de Sobolev logarithmiques gaussiennes, C. R. Acad. Sci. Paris Ser. I Math. 306 (1988), 79-92. 
[66] M. Ledoux, The Concentration of Measure Phenomenon, Math. Surveys 89, Amer. Math. Soc., 2001.

[67] M. Ledoux, From concentration to isoperimetry: Semigroup proofs, Cont. Math. 545 (2011), 155-166.

[68] G. Leoni, A first course in Sobolev spaces, Grad. Studies in Math. 105, Amer. Math. Soc. 2009.

[69] J. Malý and L. Pick, An elementary proof of Sharp Sobolev embeddings, Proc. Amer. Math. Soc. 130 (2002), 555-563.

[70] J. Martín and M. Milman, Symmetrization inequalities and Sobolev embeddings, Proc. Amer. Math. Soc. 134 (2006), 2335-2347.

[71] J. Martín and M. Milman, Higher-order symmetrization inequalities and applications, J. Math. Anal. Appl. 330 (2007), 91-113.

[72] J. Martin and M. Milman, A note on Sobolev inequalities and limits of Lorentz spaces, Contemp. Math. 445 (2007), 237-245.

[73] J. Martin and M. Milman, Isoperimetry and Symmetrization for Logarithmic Sobolev inequalities, Journal of Functional Analysis 256 (2009), $149-178$.

[74] J. Martin and M. Milman, Addendum to Isoperimetry and Symmetrization for Logarithmic Sobolev inequalities, (arXiv:0901.1839)

[75] J. Martin and M. Milman, Isoperimetry and Symmetrization for Sobolev spaces on metric spaces, Comptes Rendus Math. 347 (2009), 627-630.

[76] J. Martín and M. Milman, Isoperimetric Hardy type and Poincaré inequalities on metric spaces, In: Around the Research of Vladimir Maz'ya I. Function Spaces - Ari Laptev (Ed.) International Mathematical Series, Springer 11 (2010), 285-298.

[77] J. Martin and M. Milman, Pointwise Symmetrization Inequalities for Sobolev functions and applications, Adv. Math. 225 (2010), 121-199.

[78] J. Martin and M. Milman, Sobolev inequalities, rearrangements, isoperimetry and interpolation spaces, Contemp. Math. 545 (2011), 167193.

[79] J. Martin and M. Milman, Fractional Sobolev inequalities: symmetrization, isoperimetry and interpolation, submitted (arXiv:1205.1231).

[80] J. Martin and M. Milman, A note on Coulhon type inequalities, to appear in Proc. Amer. Math. Soc. (arXiv:1206.1584)

[81] J. Martin and M. Milman, Integral isoperimetric transference and dimensionless Sobolev inequalities, submitted (arXiv:1309.1980). 
[82] J. Martin and M. Milman, A note on iterated Sobolev inequalities involving the isoperimetric profile, preprint.

[83] J. Martin and M. Milman, On the Calderón-Maz'ya-Rubio de Francia extrapolation principle, preprint 2013.

[84] J. Martin and M. Milman, Symmetrization methods in the theory of Sobolev inequalities, Lecture Notes, in preparation.

[85] J. Martin, M. Milman and E. Pustylnik, Sobolev Inequalities: Symmetrization and Self Improvement via truncation, Journal of Functional Analysis 252 (2007), 677-695.

[86] M. Mastylo, The Modulus of Smoothness in Metric Spaces and Related Problems, Potential Anal. 35 (2011), 301-328.

[87] V. G. Maz'ya, Sobolev Spaces with applications to elliptic partial differential equations. Second, revised and augmented edition. Grundlehren der Mathematischen Wissenschaften [Fundamental Principles of Mathematical Sciences], 342. Springer, Heidelberg, 2011.

[88] V. G. Maz'ya, The p-conductivity and theorems on imbedding certain functional spaces into a $C$-space (Russian), Dokl. Akad. Nauk SSSR 140 (1961), 299-302 (English translation: in Soviet Math. Dokl. 3 (1962).

[89] E. Milman, Concentration and isoperimetry are equivalent assuming curvature lower bound, C. R. Math. Acad. Sci. Paris 347 (2009), 73-76.

[90] E. Milman, On the role of Convexity in Isoperimetry, Spectral-Gap and Concentration, Invent. Math. 177 (2009), 1-43.

[91] E. Milman, On the role of convexity in functional and isoperimetric inequalities, Proc. London Math. Soc., Proc. 999 (2009), 32-66.

[92] E. Milman, Isoperimetric and Concentration Inequalities - Equivalence under Curvature Lower Bound, Duke Math. J. 154 (2010), 207-239.

[93] E. Milman, A converse to the Maz'ya inequality for capacities under curvature lower bound, in A. Laptev (ed), Around the research of Vladimir Maz'ya I: Function Spaces, Springer, 2010, pp 321-348.

[94] E. Milman, Isoperimetric bounds on convex manifolds, Contemp. Math 545 (2011), 195-208.

[95] M. Milman, Local operators vs Lorentz-Marcinkiewicz spaces. Interpolation spaces and related topics (Haifa, 1990), Israel Math. Conf. Proc. 5 (1992), 151-157.

[96] M. Milman and E. Pustylnik, On sharp higher order Sobolev embeddings, Comm. Contemp. Math. 6 (2004), 495-511. 
[97] http://en.wikipedia.org/wiki/Vitali_Milman

[98] E. Oklander, Interpolacion, espacios de Lorentz y teorema de Marcinkiewicz, Cursos y Seminarios 20, Univ. Buenos Aires, 1965. (See also E. Oklander, On interpolation of Banach spaces, Thesis, Univ. Chicago, 1963)

[99] R. O'Neil, Convolution operators and $L(p, q)$ spaces, Duke Math. J. 30 (1963), 129-142.

[100] F.J. Pérez Lázaro, A note on extreme cases of Sobolev embeddings, J. Math. Anal. Appl. 320 (2006), 973-982.

[101] L. Pick, A. Kufner, O. John and S. Fucik, Function Spaces, Volume 1, Walter de Gruyter \& Co, Berlin, 2012

[102] G. Pisier, Factorization of operators through $L_{p \infty}$ or $L_{p 1}$ and noncommutative generalizations, Math. Ann. 276 (1986), 105-136.

[103] E. Pustylnik, On compactness of Sobolev embeddings in rearrangementinvariant spaces, Forum Math. 18 (2006), 839-852.

[104] J. M. Rakotoson, Réarrangement relatif. Un instrument d'estimations dans les problèmes aux limites, Mathematics \& Applications 64, Springer, Berlin, 2008..

[105] A. Ros, The isoperimetric problem, In: Global Theory of Minimal Surfaces. Clay Math. Proc., vol. 2, pp. 175-209, Am. Math. Soc., Providence, 2005

[106] G. C. Rota, Ten Lessons I wish I had been Taught, (http://alumni.media.mit.edu/ cahn/life/gian-carlo-rota-10-lessons.html)

[107] L. Saloff-Coste, Aspects of Sobolev inequalities, Cambridge University Press, 2002.

[108] L. Slavíková, Compactness of higher order Sobolev embeddings, Master Thesis, Charles University, Prague 2012.

[109] V. N. Sudakov and B. S. Tsirelson, Extremal properties of half-spaces for spherically invariant measures. J. Soviet. Math. 9 (1978), 918; translated from Zap. Nauch. Sem. L.O.M.I. 41 (1974), 1424.

[110] G. Talenti, Inequalities in rearrangement-invariant function spaces, in: Nonlinear Analysis, Function Spaces and Applications, vol. 5, Prometheus, Prague, 1995, pp. 177-230. for a comprehensive bibliography

[111] L. Tartar, Imbedding theorems of Sobolev spaces into Lorentz spaces, Boll. Unione Mat. Ital. Sez B Artic. Ric. Mat. (8) 1 (1998), 479-500. 
[112] N. Trudinger, On imbeddings into Orlicz spaces and some applications, J. Math. Mech. 17 (1967) 473-483.

[113] H. Triebel, Tractable embeddings of Besov spaces into Zygmund spaces, Function spaces IX, 361-377, Banach Center Publ. 92, Polish Acad. Sci. Inst. Math., Warsaw, 2011.

[114] H. Triebel, Tractable embeddings, preprint, University of Jena, Nov. 2012.

[115] J. Xiao and Z. Zhai, Fractional Sobolev, Moser-Trudinger, Morrey-Sobolev inequalities under Lorentz norms, J. Math. Sci. (New York), 166 (2010), $357-376$. 